\title{
Thermohaline instability and rotation-induced mixing
}

\section{Grid of stellar models and asymptotic asteroseismic quantities from the pre-main sequence up to the AGB for low- and intermediate-mass stars of various metallicities ${ }^{\star}$}

\author{
N. Lagarde ${ }^{1}$, T. Decressin ${ }^{1}$, C. Charbonnel ${ }^{1,2}$, P. Eggenberger ${ }^{1}$, S. Ekström ${ }^{1}$, and A. Palacios ${ }^{3}$ \\ 1 Geneva Observatory, University of Geneva, Chemin des Maillettes 51, 1290 Versoix, Switzerland \\ e-mail: Nadege.Lagarde@unige.ch \\ 2 IRAP, UMR 5277 CNRS and Université de Toulouse, 14 Av. E. Belin, 31400 Toulouse, France \\ 3 LUPM, Université Montpellier II, CNRS, UMR 5299, Place E. Bataillon, 34095 Montpellier, France
}

Received 25 October 2011 / Accepted 13 April 2012

\section{ABSTRACT}

\begin{abstract}
Context. The availability of asteroseismic constraints for a large sample of stars from the missions CoRoT and Kepler paves the way for various statistical studies of the seismic properties of stellar populations.

Aims. We evaluate the impact of rotation-induced mixing and thermohaline instability on the global asteroseismic parameters at different stages of the stellar evolution from the zero age main sequence to the thermally pulsating asymptotic giant branch to distinguish stellar populations.

Methods. We present a grid of stellar evolutionary models for four metallicities $(Z=0.0001,0.002,0.004$, and 0.014$)$ in the mass range from 0.85 to $6.0 M_{\odot}$. The models are computed either with standard prescriptions or including both thermohaline convection and rotation-induced mixing. For the whole grid, we provide the usual stellar parameters (luminosity, effective temperature, lifetimes, ... ), together with the global seismic parameters, i.e. the large frequency separation and asymptotic relations, the frequency corresponding to the maximum oscillation power $v_{\max }$, the maximal amplitude $A_{\max }$, the asymptotic period spacing of g-modes, and different acoustic radii.

Results. We discuss a signature of rotation-induced mixing on the global asteroseismic quantities, that can be detected observationally. Thermohaline mixing whose effects can be identified using spectroscopic studies cannot be characterized by the global seismic parameters studied here. However, we cannot exclude that individual mode frequencies or other well chosen asteroseismic quantities might help us to constrain this mixing.
\end{abstract}

Key words. asteroseismology - instabilities - stars: evolution - stars: interiors - stars: rotation

\section{Introduction}

Much effort has been devoted to improving our understanding of the physics of low- and intermediate-mass stars, and in particular explaining the abundance anomalies they exhibit during their lifetime. Rotation has been shown to change the internal dynamics of these stars, by means of the transport of both angular momentum and chemical species through the action of meridional circulation and shear turbulence, combined possibly with other processes induced by internal gravity waves or magnetic fields (see e.g. Zahn 1992; Zahn et al. 1997; Maeder \& Zahn 1998; Talon \& Charbonnel 1998; Eggenberger et al. 2005; Charbonnel $\&$ Talon 2005, 2008). Rotation-induced mixing results in variations of the stellar chemical properties that successfully explain many of the abundance patterns observed at the surface of these stars (Palacios et al. 2003; Charbonnel \& Talon 2008; Smiljanic et al. 2010; Charbonnel \& Lagarde 2010). In addition, thermohaline mixing driven by ${ }^{3} \mathrm{He}$-burning has been proposed to be the most likely process modifying the photospheric compositions of bright low-mass red giant stars (Charbonnel \& Zahn 2007b; for references on the abundance anomalies at that evolution

* Results tables are only available at the CDS via anonymous ftp to cdsarc.u-strasbg.fr (130.79.128.5) or via

http://cdsarc.u-strasbg.fr/viz-bin/qcat?J/A+A/543/A108 phase, see Charbonnel \& Lagarde 2010, hereafter Paper I; and Lagarde et al. 2011, hereafter Paper II). During the thermalpulse phase on the asymptotic giant branch (TP-AGB), thermohaline mixing was found to lead to lithium production (Paper I; Stancliffe 2010), accounting for the Li abundances observed in oxygen-rich AGB variables of the Galactic disk (Uttenthaler \& Lebzelter 2010). In summary and as discussed in Papers I and II of this series (see also Charbonnel \& Zahn 2007b), the effects of both rotation-induced mixing and thermohaline instability as described presently do account very nicely for most of the spectroscopic observations of low- and intermediate-mass stars at various metallicities.

This has crucial consequences for the chemical evolution of the Galaxy (Paper II, and Lagarde et al., in prep.), and should also be taken into account in the other topical astrophysical domains that use stellar models as input physics. This is particuliarly true as asteroseismic probes observe stars across the Hertzsprung-Russell (HR) diagram. Thanks to the development of dedicated satellites such as CoRoT and Kepler, the internal properties of stars on both the main sequence (e.g. Michel et al. 2008; Chaplin et al. 2010) and the giant branches (e.g. De Ridder et al. 2009; Bedding et al. 2010) have been revealed. Furthermore, owing to the large number of stars observed by these missions, statistical studies are possible through 
the determination of global pulsation properties such as the frequency of maximum oscillation power and the large frequency separation (see e.g. Miglio et al. 2009; Chaplin et al. 2011a).

In this broad context, the aim of the present paper is to provide the relevant classical stellar parameters together with the global asteroseismic properties of low- and intermediate-mass stars throughout their evolution. This is done from the pre-main sequence (along the Hayashi track) to the early-AGB (and along the TP-AGB for selected cases) for the grid of models computed in Papers I and II for four metallicities spanning the range between $Z=0.0001$ and $Z=0.014$ and with initial masses between $0.85 M_{\odot}$ and $6.0 M_{\odot}$. This grid contains models computed with rotation-induced mixing and thermohaline instability, along with for comparison purposes standard models without mixing outside convective regions. The present work is a prerequisite to validating the current theoretical prescriptions for the nonstandard mechanisms before we test them through detailed seismic analysis of individual stars.

Such a grid of stellar models at different metallicities is obviously a key tool for various important astrophysical topics related to e.g., stellar evolution in clusters, stellar nucleosynthesis, and chemical evolution. They have been available for a long time as part of standard stellar models (e.g. Schaller et al. 1992; Forestini \& Charbonnel 1997; Yi et al. 2003; Cassisi et al. 2006), and have more recently appeared in the literature for rotation-induced models (Brott et al. 2011; Ekström et al. 2012). However, these latest studies focus more on the evolution of massive stars, and neitherinclude thermohaline mixing nor study the TP-AGB phase for low- and intermediate-mass stars as we do here.

The paper is organized as follows. In Sect. 2, we describe the physical inputs of the stellar evolution models. The table content of our grids is presented in Sect. 3. Section 4 includes a short discussion on the main properties of the models and a comparison with the solar metallicity models of Ekström et al. (2012). In Sect. 5, we present the asteroseismic parameters of models with and without rotation. Finally, our main results are summarized in Sect. 6.

\section{Physical inputs}

The models are computed with the implicit Lagrangian stellarevolution code STAREVOL (v3.00. See Siess et al. 2000; Palacios et al. 2003, 2006; Decressin et al. 2009). In this section, we summarize the main physical ingredients used for the present grid.

\subsection{Basic inputs}

The description of the stellar structure rests on the hydrostatic and the continuity equations, and the equations for energy conservation and transport. To solve this system, the following physical ingredients are required:

- Nuclear reaction rates are needed to follow the chemical changes inside burning sites, and to determine the production of energy by the nuclear reaction, $\epsilon_{\text {nuc }}$, and the energy loss by the neutrino, $\epsilon_{v}$. We follow stellar nucleosynthesis with a network including 185 nuclear reactions involving 54 stable and unstable species from ${ }^{1} \mathrm{H}$ to ${ }^{37} \mathrm{Cl}$. Numerical tables for the nuclear reaction rates were generated from the NACRE compilation (Arnould et al. 1999; Aikawa et al. 2005) with the NetGen web interface ${ }^{1}$.

${ }^{1}$ http://www.astro.ulb.ac.be/Netgen/form.html
We mainly use reactions rates from either NACRE or Caughlan \& Fowler (1988) when NACRE rates are unavailable. For proton captures on elements higher then $\mathrm{Ne}$, we follow rates from Iliadis et al. (2001) or otherwise Bao et al. (2000). The following reactions rates are computed by:

- ${ }^{3} \mathrm{He}(\mathrm{D}, \mathrm{p}){ }^{4} \mathrm{He}$ (Descouvemont et al. 2004);

$-{ }^{3} \mathrm{He}(\alpha \alpha, \gamma){ }^{12} \mathrm{C}$ (Fynbo et al. 2005);

- ${ }^{8} \mathrm{~B}(\beta, v) 2{ }^{4} \mathrm{He} ;{ }^{13} \mathrm{~N}(\beta, v){ }^{13} \mathrm{C} ;{ }^{22} \mathrm{Na}(\beta, v){ }^{22} \mathrm{Ne} ;{ }^{26} \mathrm{Alm}(\beta$, $v)^{26} \mathrm{Mg} ;{ }^{26} \mathrm{Alg}(\beta, v){ }^{26} \mathrm{Mg}$ (Horiguchi et al. 1996);

- ${ }^{14} \mathrm{C}(\mathrm{p}, \gamma){ }^{15} \mathrm{~N}$ (Wiescher et al. 1990);

- ${ }^{14} \mathrm{C}(\mathrm{p}, \mathrm{n}){ }^{14} \mathrm{~N}$ (Koehler \& O’brien 1989a);

- ${ }^{14} \mathrm{C}(\alpha, \mathrm{n}){ }^{17} \mathrm{O} ;{ }^{17} \mathrm{O}\left(\mathrm{n},{ }^{4} \mathrm{He}\right){ }^{14} \mathrm{C}$ (Schatz et al. 1993);

- ${ }^{14} \mathrm{C}(\alpha, \gamma){ }^{18} \mathrm{O}$ (Funck \& Langanke 1989);

- ${ }^{14} \mathrm{~N}(\mathrm{n}, \mathrm{p}){ }^{14} \mathrm{C}$ (Koehler \& O'brien 1989b);

- ${ }^{14} \mathrm{~N}(\mathrm{p}, \gamma){ }^{15} \mathrm{O}$ (Mukhamedzhanov et al. 2003);

- ${ }^{17} \mathrm{O}(\mathrm{n}, \gamma){ }^{18} \mathrm{O}$ (Wagoner 1969);

- ${ }^{22} \mathrm{Ne}(\mathrm{p}, \gamma){ }^{23} \mathrm{Na}$ (Hale et al. 2002);

- ${ }^{22} \mathrm{Ne}(\mathrm{n}, \gamma)^{23} \mathrm{Na}$ (Beer et al. 2002);

- ${ }^{22} \mathrm{Na}(\mathrm{n}, \quad \gamma)^{23} \mathrm{Na} ;{ }^{23} \mathrm{Na}(\alpha, \quad \mathrm{p}){ }^{26} \mathrm{Mg} ;{ }^{25} \mathrm{Mg}(\alpha, \quad \mathrm{p}){ }^{28} \mathrm{Si}$; ${ }^{26} \mathrm{Mg}(\alpha, \mathrm{p}){ }^{29} \mathrm{Si} ;{ }^{27} \mathrm{Al}(\alpha, \mathrm{p}){ }^{30} \mathrm{Si}$ (Hauser \& Feshbach 1952);

- ${ }^{26} \mathrm{Alm}(\mathrm{n}, \gamma)^{27} \mathrm{Al} ;{ }^{26} \mathrm{Alg}(\mathrm{n}, \gamma)^{27} \mathrm{Al}$ (Woosley et al. 1978) ${ }^{2}$.

- The screening factors are calculated with the formalism of Mitler (1977) for weak and intermediate screening conditions and Graboske et al. (1973) for strong screening conditions.

- Opacities are required to compute the radiative gradient $\nabla_{\text {rad }}$ and the energy transport by radiative transfer. We generate opacity tables according to Iglesias \& Rogers (1996) using the OPAL website ${ }^{3}$ for $T>8000 \mathrm{~K}$ that account for $\mathrm{C}$ and $\mathrm{O}$ enrichments. At lower temperature $(T<8000 \mathrm{~K})$, we use the atomic and molecular opacities given by Ferguson et al. (2005).

- The equation of state relates the temperature, pressure, and density and thus provides different thermodynamic quantities $\left(\nabla_{\mathrm{ad}}, c_{\mathrm{P}}, \ldots\right)$. In STAREVOL, we follow the formalism developed by Eggleton et al. (1973) and extended by Pols et al. (1995), which is based on the principle of Helmholtz free energy minimization (see Dufour 1999; and Siess et al. 2000, for detailed description and numerical implementation): this accounts for the non-ideal effects of Coulomb interactions and pressure ionization.

- The treatment of convection is needed to compute the temperature gradient inside a convective zone. It is based on a classical mixing-length formalism with $\alpha_{\mathrm{MLT}}=1.6$, recovered from solar-calibrated models that include neither atomic diffusion nor rotation and were computed using Geneva models (see Ekström et al. 2012). We assume instantaneous convective mixing, except when hot-bottom burning occurs on the TP-AGB, which requires a time-dependent convective diffusion algorithm as developed in Forestini \& Charbonnel (1997). The boundary between convective and radiative layers is defined with the Schwarzschild criterion. An overshoot parameter $d_{\text {over }} / H_{\mathrm{p}}$ is taken into account for the convective core. This parameter is set to 0.05 or 0.10 respectively for stars with masses below or above $2.0 M_{\odot}{ }^{4}$.

- We use a gray atmosphere where the photosphere is defined as the layer for which the optical depth $\tau$ is between 0.005

\footnotetext{
$\overline{{ }^{2}{ }^{26} \mathrm{Alm} \text { and }{ }^{26} \mathrm{Alg} \text { represent }}$ the radioactive nuclide ${ }^{26} \mathrm{Al}$ in its two isomeric states.

${ }^{3}$ http://adg.1lnl.gov/Research/OPAL/opal.html

${ }^{4}$ For small cores its mass is not allowed to be higher than $d_{\text {over }}$ times the core mass.
} 
and 10 . We define the effective temperature and radius at the layer where $\tau=2 / 3$.

- For mass loss, we use Reimers (1975) formula (with $\eta_{R}=$ $0.5)$ from the ZAMS up to central helium exhaustion

$\dot{M}=-3.9810^{-13} \eta_{R} \frac{L R}{M} M_{\odot} \mathrm{yr}^{-1}$.

On the AGB, we adopt the mass-loss prescription of Vassiliadis \& Wood (1993).

\subsection{Transport processes in radiative zones}

\subsubsection{Thermohaline mixing}

Thermohaline instability develops along the red giant branch (RGB) at the bump luminosity in low-mass stars and on the early-AGB in intermediate-mass stars, when the gradient of molecular weight becomes negative $\left(\nabla_{\mu}=\frac{\operatorname{dln} \mu}{\operatorname{dn} P}<0\right)$ in the external wing of the thin hydrogen-burning shell surrounding the degenerate stellar core (Charbonnel \& Zahn 2007b,a; Siess 2009; Stancliffe et al. 2009; Charbonnel \& Lagarde 2010). This inversion of molecular weight is created by the ${ }^{3} \mathrm{He}\left({ }^{3} \mathrm{He}, 2 \mathrm{p}\right){ }^{4} \mathrm{He}$ reaction (Ulrich 1971; Eggleton et al. 2006, 2008).

The present grid of models is computed using the prescription advocated by Charbonnel \& Zahn (2007b) and Papers I, II. It is based on Ulrich (1972) with an aspect ratio of instability fingers $\alpha=6$, in agreement with laboratory experiments (Krishnamurti 2003). It includes the correction for non-perfect gas (including radiation pressure, degeneracy) in the diffusion coefficient for thermohaline mixing that is given by:

$D_{\mathrm{t}}=C_{\mathrm{t}} K\left(\frac{\varphi}{\delta}\right) \frac{-\nabla_{\mu}}{\left(\nabla_{\mathrm{ad}}-\nabla\right)} \quad$ for $\nabla_{\mu}<0$,

where $K$ is the thermal diffusivity; $\varphi=(\partial \ln \rho / \partial \ln \mu)_{P, T} ; \delta=$ $-(\partial \ln \rho / \partial \ln v)_{P, \mu}$; and with the non-dimensional coefficient

$C_{\mathrm{t}}=\frac{8}{3} \pi^{2} \alpha^{2}$.

The value of $\alpha$ in actual stellar conditions was recently questionned by the results of two- and three-dimentional hydrodynamical simulations of thermohaline convection for which $\alpha$ is close to unity (Denissenkov 2010; Denissenkov \& Merryfield 2011; Rosenblum et al. 2011; Traxler et al. 2011). However, these simulations are still far from the stellar regime, hence we decided to use in this series the prescription described above since it successfully reproduces the abundance data for evolved stars of various masses and metallicities (see Papers I and II for a more detailed discussion).

\subsubsection{Rotation-induced mixing}

Pre-main sequence evolution along the Hayashi track is computed in a standard way (i.e., without accounting for rotationinduced mixing), and solid-body rotation is assumed on the Zero Age Main Sequence (ZAMS). On the main sequence, the evolution of the internal angular momentum profile is accounted for using the complete formalism developed by Zahn (1992), Maeder \& Zahn (1998), and Mathis \& Zahn (2004), which takes into account advection by meridional circulation and diffusion by shear turbulence (see Palacios et al. 2003, 2006; Decressin et al. 2009, for a description of the implementation in STAREVOL). We do not take into account the inhibitory effects of $\mu$ gradients in the treatement of rotation.
We assume solid-body rotation in the convective regions, as we did in Papers I and II. In addition, we assume that the transport of angular momentum is dominated by the large amount of turbulence in these regions that instantaneously flattens out the angular velocity profile as it does for the abundance profiles. This hypothesis leads to a minimum shear-mixing approach in the underlying radiative layers, as discussed in Palacios et al. (2006) and Brun \& Palacios (2009).

On the other hand, the transport of angular momentum in stellar radiative layers obeys the advection/diffusion equation

$\rho \frac{\mathrm{d}\left(r^{2} \Omega\right)}{\mathrm{d} t}=\frac{1}{5 r^{2}} \frac{\partial}{\partial r}\left(\rho r^{4} \Omega U_{r}\right)+\frac{1}{r} \frac{\partial}{\partial r}\left(r^{4} \rho v_{v} \frac{\partial \Omega}{\partial r}\right)$

where $\rho, r$, and $\Omega$ have their usual meaning, $U_{r}$ is the vertical component of meridional circulation velocity, and $v_{v}$ is the vertical component of the turbulence viscosity.

The transport of chemical species resulting from meridional circulation and both vertical and horizontal turbulence is computed as a diffusive process (Chaboyer \& Zahn 1992). The vertical transport of a chemical species $i$ of concentration $c_{i}$ is described by a pure diffusion equation

$$
\frac{\mathrm{d} c_{i}}{\mathrm{~d} t}=\underbrace{\dot{c}_{i}}_{\text {nuclear }}+\underbrace{\frac{1}{\rho r^{2}} \frac{\partial}{\partial r}\left(r^{2} \rho D_{\mathrm{tot}} \frac{\partial c_{i}}{\partial r}\right)}_{\text {diffusion processes }},
$$

where $\dot{c}_{i}$ represents the variations in the chemical composition due to nuclear reactions. The total diffusion coefficient $D_{\text {tot }}$ for chemicals can be written as the sum of three coefficients

$D_{\text {tot }}=D_{\text {th }}+D_{\text {eff }}+D_{\mathrm{v}}$

with $D_{\text {th }}$ the thermohaline coefficient (Sect. 2.2.1), $D_{\text {eff }}$ the effective diffusion coefficient $\left(\propto 1 / D_{\mathrm{h}}\right)$, and $D_{\mathrm{v}}$ the vertical turbulent diffusion coefficient (Talon \& Zahn 1997), the last of which is proportional to the horizontal diffusion coefficient Chaboyer \& Zahn 1992). The expression of the horizontal diffusion coefficient is taken from Zahn (1992), with its expression that prevents numerical divergence

$D_{\mathrm{h}}=\frac{r}{C_{\mathrm{h}}}\left(|2 V-\alpha U|^{2}+U^{2}\right)^{1 / 2}$.

We do consider neither possible interactions between thermohaline and rotation-induced mixing, nor magnetic diffusion. Under the present assumptions, the thermohaline diffusion coefficient is several orders of magnitude higher than both the total diffusion coefficient related to rotation and the magnetic diffusivity in the advanced phases where the thermohaline instability develops (see e.g., Charbonnel \& Lagarde 2010; Cantiello \& Langer 2010, and Paper II).

The complete treatment is applied up to either the RGB tip or the second dredge-up for stars undergoing the helium-flash episode.

\subsection{Initial rotation velocity}

The initial rotation velocity of our models on the ZAMS is chosen at $45 \%$ of the critical velocity at that point, with $V_{\text {crit }}=$ $\left(\frac{2}{3}\right)^{\frac{3}{2}}\left(\frac{G M}{R}\right)^{\frac{1}{2}}$. Here we take $R$ to be the stellar radius computed without considering the stellar deformation due to rotation; if we were to take into account the deformation of the stellar radius as in Ekström et al. (2012), then our initial velocities would correspond to $30 \%$ of the critical velocity. This choice 
Table 1. Initial abundances in mass fraction for the models at different metallicities.

\begin{tabular}{|c|c|c|c|c|}
\hline $\begin{array}{l}{[\mathrm{Fe} / \mathrm{H}]} \\
\text { Element }\end{array}$ & $\begin{array}{c}0 \\
Z=0.014\end{array}$ & $\begin{array}{c}-0.56 \\
Z=0.004\end{array}$ & $\begin{array}{c}-0.86 \\
Z=0.002\end{array}$ & $\begin{array}{c}-2.16 \\
Z=0.0001\end{array}$ \\
\hline${ }^{1} \mathrm{H}$ & $7.20 \times 10^{-01}$ & $7.42 \times 10^{-01}$ & $7.47 \times 10^{-01}$ & $7.52 \times 10^{-01}$ \\
\hline${ }^{2} \mathrm{H}$ & $3.74 \times 10^{-05}$ & $3.86 \times 10^{-05}$ & $3.89 \times 10^{-05}$ & $3.91 \times 10^{-05}$ \\
\hline${ }^{3} \mathrm{He}$ & $2.83 \times 10^{-05}$ & $2.69 \times 10^{-05}$ & $2.67 \times 10^{-05}$ & $2.64 \times 10^{-05}$ \\
\hline${ }^{4} \mathrm{He}$ & $2.66 \times 10^{-01}$ & $2.53 \times 10^{-01}$ & $2.50 \times 10^{-01}$ & $2.48 \times 10^{-01}$ \\
\hline${ }^{6} \mathrm{Li}$ & $6.35 \times 10^{-10}$ & $1.81 \times 10^{-10}$ & $9.07 \times 10^{-11}$ & $4.53 \times 10^{-12}$ \\
\hline${ }^{7} \mathrm{Li}$ & $9.00 \times 10^{-09}$ & $2.16 \times 10^{-09}$ & $2.17 \times 10^{-09}$ & $2.18 \times 10^{-09}$ \\
\hline${ }^{9} \mathrm{Be}$ & $1.69 \times 10^{-10}$ & $4.82 \times 10^{-11}$ & $2.41 \times 10^{-11}$ & $1.21 \times 10^{-12}$ \\
\hline${ }^{10} \mathrm{~B}$ & $8.09 \times 10^{-10}$ & $2.31 \times 10^{-10}$ & $1.16 \times 10^{-10}$ & $5.78 \times 10^{-12}$ \\
\hline${ }^{11} \mathrm{~B}$ & $3.94 \times 10^{-09}$ & $1.13 \times 10^{-09}$ & $5.63 \times 10^{-10}$ & $2.82 \times 10^{-11}$ \\
\hline${ }^{12} \mathrm{C}$ & $2.27 \times 10^{-03}$ & $6.47 \times 10^{-04}$ & $3.24 \times 10^{-04}$ & $1.62 \times 10^{-05}$ \\
\hline${ }^{13} \mathrm{C}$ & $3.63 \times 10^{-05}$ & $1.04 \times 10^{-05}$ & $5.19 \times 10^{-06}$ & $2.59 \times 10^{-07}$ \\
\hline${ }^{14} \mathrm{~N}$ & $6.56 \times 10^{-04}$ & $1.87 \times 10^{-04}$ & $9.38 \times 10^{-05}$ & $4.69 \times 10^{-06}$ \\
\hline${ }^{15} \mathrm{~N}$ & $2.34 \times 10^{-06}$ & $6.69 \times 10^{-07}$ & $3.35 \times 10^{-07}$ & $1.67 \times 10^{-08}$ \\
\hline${ }^{16} \mathrm{O}$ & $5.69 \times 10^{-03}$ & $1.62 \times 10^{-03}$ & $8.13 \times 10^{-04}$ & $4.06 \times 10^{-05}$ \\
\hline${ }^{17} \mathrm{O}$ & $3.82 \times 10^{-06}$ & $1.09 \times 10^{-06}$ & $5.46 \times 10^{-07}$ & $2.73 \times 10^{-08}$ \\
\hline${ }^{18} \mathrm{O}$ & $1.28 \times 10^{-05}$ & $3.67 \times 10^{-06}$ & $1.83 \times 10^{-06}$ & $9.17 \times 10^{-08}$ \\
\hline${ }^{19} \mathrm{~F}$ & $5.38 \times 10^{-07}$ & $1.54 \times 10^{-07}$ & $7.69 \times 10^{-08}$ & $3.85 \times 10^{-09}$ \\
\hline${ }^{20} \mathrm{Ne}$ & $1.79 \times 10^{-03}$ & $5.10 \times 10^{-04}$ & $2.55 \times 10^{-04}$ & $1.28 \times 10^{-05}$ \\
\hline${ }^{21} \mathrm{Ne}$ & $5.70 \times 10^{-06}$ & $1.63 \times 10^{-06}$ & $8.14 \times 10^{-07}$ & $4.07 \times 10^{-08}$ \\
\hline${ }^{22} \mathrm{Ne}$ & $2.40 \times 10^{-04}$ & $6.85 \times 10^{-05}$ & $3.42 \times 10^{-05}$ & $1.71 \times 10^{-06}$ \\
\hline${ }^{23} \mathrm{Na}$ & $2.65 \times 10^{-05}$ & $7.58 \times 10^{-06}$ & $3.79 \times 10^{-06}$ & $1.89 \times 10^{-07}$ \\
\hline${ }^{24} \mathrm{Mg}$ & $4.99 \times 10^{-04}$ & $1.42 \times 10^{-04}$ & $7.13 \times 10^{-05}$ & $3.57 \times 10^{-06}$ \\
\hline${ }^{25} \mathrm{Mg}$ & $6.69 \times 10^{-05}$ & $1.91 \times 10^{-05}$ & $9.56 \times 10^{-06}$ & $4.78 \times 10^{-07}$ \\
\hline${ }^{26} \mathrm{Mg}$ & $7.67 \times 10^{-05}$ & $2.19 \times 10^{-05}$ & $1.10 \times 10^{-05}$ & $5.48 \times 10^{-07}$ \\
\hline${ }^{27} \mathrm{Al}$ & $4.94 \times 10^{-05}$ & $1.41 \times 10^{-05}$ & $7.05 \times 10^{-06}$ & $3.52 \times 10^{-07}$ \\
\hline${ }^{28} \mathrm{Si}$ & $5.97 \times 10^{-04}$ & $1.71 \times 10^{-04}$ & $8.53 \times 10^{-05}$ & $4.27 \times 10^{-06}$ \\
\hline${ }^{29} \mathrm{Si}$ & $6.65 \times 10^{-05}$ & $1.90 \times 10^{-05}$ & $9.50 \times 10^{-06}$ & $4.75 \times 10^{-07}$ \\
\hline${ }^{30} \mathrm{Si}$ & $4.81 \times 10^{-05}$ & $1.38 \times 10^{-05}$ & $6.87 \times 10^{-06}$ & $3.44 \times 10^{-07}$ \\
\hline${ }^{31} \mathrm{P}$ & $5.54 \times 10^{-06}$ & $1.58 \times 10^{-06}$ & $7.91 \times 10^{-07}$ & $3.96 \times 10^{-08}$ \\
\hline${ }^{32} \mathrm{~S}$ & $3.24 \times 10^{-04}$ & $9.26 \times 10^{-05}$ & $4.63 \times 10^{-05}$ & $2.31 \times 10^{-06}$ \\
\hline${ }^{33} \mathrm{~S}$ & $3.48 \times 10^{-06}$ & $9.95 \times 10^{-07}$ & $4.97 \times 10^{-07}$ & $2.49 \times 10^{-08}$ \\
\hline${ }^{34} \mathrm{~S}$ & $1.80 \times 10^{-05}$ & $5.15 \times 10^{-06}$ & $2.58 \times 10^{-06}$ & $1.29 \times 10^{-07}$ \\
\hline${ }^{35} \mathrm{Cl}$ & $6.54 \times 10^{-06}$ & $1.87 \times 10^{-06}$ & $9.34 \times 10^{-07}$ & $4.67 \times 10^{-08}$ \\
\hline${ }^{37} \mathrm{Cl}$ & $2.15 \times 10^{-06}$ & $6.14 \times 10^{-07}$ & $3.07 \times 10^{-07}$ & $1.53 \times 10^{-08}$ \\
\hline Others & $1.47 \times 10^{-03}$ & $4.19 \times 10^{-04}$ & $2.09 \times 10^{-04}$ & $1.05 \times 10^{-05}$ \\
\hline
\end{tabular}

of $V_{\text {ini }} / V_{\text {crit }}=0.45$ closely agrees with the mean value of the observed velocity distribution for low- and intermediate-mass stars in young open clusters. This initial rotation rate leads to mean velocities on the main sequence of between 90 and $137 \mathrm{~km} \mathrm{~s}^{-1}$.

We apply magnetic braking only to $\left[1.25 M_{\odot} ; Z_{\odot}\right]$ and [0.85 $\left.M_{\odot} ; Z=0.0001\right]$ following the description of Kawaler (1988) according to Talon \& Charbonnel (1998) and Charbonnel \& Talon (1999).

We neglect the transport by internal gravity waves, which is efficient only in main sequence stars with effective temperatures on the ZAMS lower than $6500 \mathrm{~K}$ (see Talon \& Charbonnel 2003). We similarly account for neither dynamo processes nor the presence of fossil magnetic fields.

\subsection{Initial abundances}

Table 1 presents the initial abundances in mass fraction that we assume at different metallicities. We adopt the solar mixture of Asplund et al. (2009), except for $\mathrm{Ne}$ for which we use the value derived by Cunha et al. (2006). We use the ratio $\Delta Y / \Delta Z=1.29$ derived by Ekström et al. (2012) to account for the enrichment in

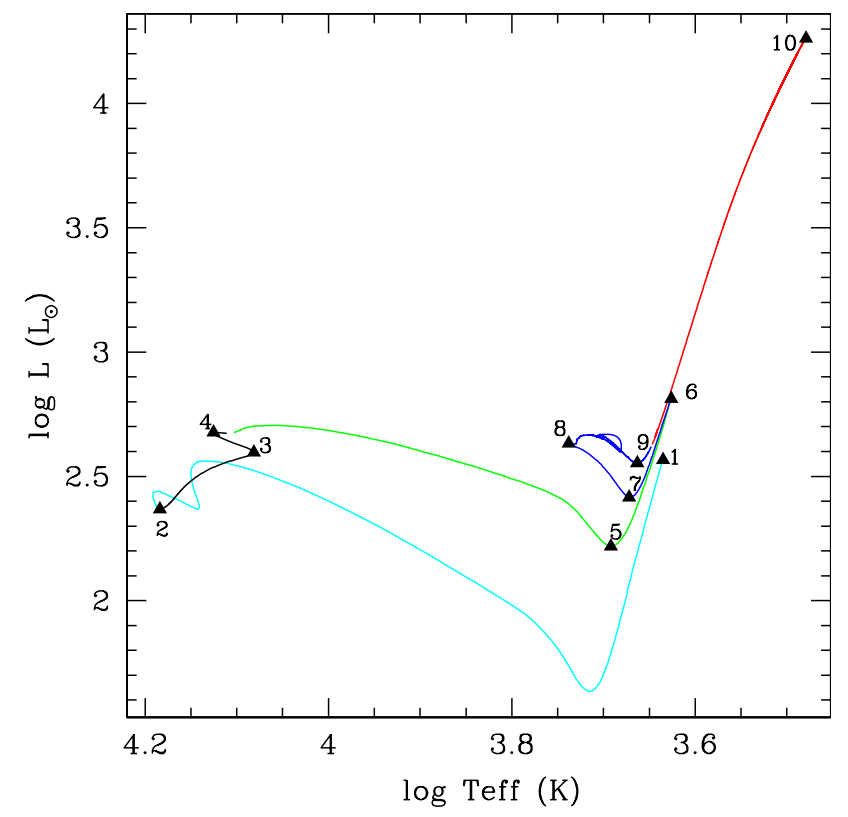

Fig. 1. Evolution track in the Hertzsprung-Russell diagram of the standard 4.0 $M_{\odot}$ model at solar metallicity. Each phase is indicated by a different color: pre-main sequence (cyan), main sequence (black), Hertzsprung gap and red giant branch (green), core helium-burning phase (blue), and asymptotic giant branch (red). Black triangles show the points that we have selected to describe the tracks (see Sect. 3.1)

helium reported to enrichment in heavy elements in the Galaxy until the birth of the Sun. For the primordial abundances, we take the WMAP-SBBN value from Coc et al. (2004). To determine the initial composition of our models at a given metallicity $Z$, we use the scaling $\left(X_{i}=X_{i, \odot} \cdot \frac{Z}{Z_{\odot}}\right)$ for all elements except for ${ }^{7} \mathrm{Li}$, which is assumed to be constant $\left(\mathrm{Li} / \mathrm{H}=4.15 \times 10^{-10}\right)$ for $\frac{Z}{X}<$ $7.8 \times 10^{-3}$. We assume that $[\alpha / \mathrm{Fe}]=0$ at all metallicities, which has a negligible impact in the present context ${ }^{5}$.

\section{Description of grids}

\subsection{Content of electronic tables}

We provide files containing sets of relevant physical quantities as a function of time that characterize our models computed in the initial mass range between $0.85 M_{\odot}$ and $6.0 M_{\odot}$ with four metallicities $Z=0.0001,0.002,0.004$, and $0.014([\mathrm{Fe} / \mathrm{H}]=-2.16$, $-0.86,-0.56$, and 0 , respectively). For each mass and metallicity, models are computed with standard prescriptions (no mixing other than that caused by convection), and for both thermohaline instability and rotational transport. For all masses, the evolution is followed from the beginning of the pre-main sequence (along the Hayashi track) up to the early-AGB phase. For each model, we selecte 500 points to allow a good description of the full raw tracks. We first established several key evolutionary points (see Fig. 1):

\section{1. beginning of the pre-main sequence;}

5 Using $[\alpha / \mathrm{Fe}]=+0.3$ instead of 0 for our $\left[1.5 M_{\odot} ; Z=0.0001\right]$ model leads to a decrease in the main-sequence lifetime by $\sim 2 \%$, and lowers the turnoff luminosity and effective temperature by $5 \%$ and $1 \%$ respectively. This is negligible compared to the effects of rotation. 
2. the ZAMS defined as the time when the central hydrogen abundance $X_{\mathrm{c}}$ has decreased by 0.003 in mass fraction compared to its initial value;

3 . turning point with the lowest $T_{\text {eff }}$ on the main sequence;

4. end of core H-burning defined as the point beyond which $X_{\mathrm{c}}$ is smaller than $10^{-7}$;

5. bottom of the red giant branch (RGB);

6. the RGB-tip;

7. local minimum of luminosity during central helium-burning;

8. local maximum of $T_{\text {eff }}$ during central helium-burning;

9. bottom of AGB: point with local minimum of luminosity after the loop on $\mathrm{HB}$;

10. end of core helium-burning defined as the point beyond which $Y_{\mathrm{c}}$ is smaller than $10^{-4}$.

The data in the tables are linearly interpolated within the results of each evolutionary model. We perform the interpolation as a function of time, of the central mass fraction of hydrogen or helium, or luminosity according to the evolutionary phase, and the final interpolated values are given at 499 points that we distribute as follows:

- 99 points evenly distributed in time that sample the pre-main sequence between points number 1 and 2;

- 110 points evenly distributed in terms of the central hydrogen mass fraction $X_{\mathrm{C}}$ that sample the main sequence with 85 points distributed between points number 2 and 3 , and 25 points distributed between points number 3 and 4 ;

- 60 points evenly spaced in time sample the Herzsprung gap between points number 4 and 5 ;

- 80 points evenly distributed in terms of $\log L$ sample the RGB between points number 5 and 6 ;

- 20, 70 and 70 points evenly distributed in terms of $Y_{C}$ respectively sample the central He burning phase between points number 6 and $7^{6}, 7$ and 8 and, 8 and 9;

- finally, 50 points evenly spaced in $\log L$ are selected between points number 9 and 10 .

For each model, we store the quantities given in Table 2 in a file that can be retrieved on the web page ${ }^{7}$.

\subsection{Comparisons between the models of Ekström et al. (2012, Geneva code) and our models (STAREVOL)}

Ekström et al. (2012) computed a large grid of models taking into account rotation for stars of mass from 0.8 to $120 M_{\odot}$ at solar metallicity with the Geneva stellar evolution code. The present grid is complementary to these models since the lowmass star models of Ekström et al. (2012) are computed only until the helium flash at the RGB tip and do not include thermohaline mixing. In addition, we presently explore asteroseismic diagnostics during the TP-AGB.

To offer this complementarity between the models of these two sets of giants, we were careful in choosing the same input physics and assumptions, although some differences remain that we describe below. Both codes adopt the same physical inputs for convection (Schwarzschild criterion and overshoot), opacities, mass loss, and nuclear reaction rates, and for the mass domain explored the different equations of state have a negligible impact on the stellar structures. The initial abundances of

\footnotetext{
${ }^{6}$ For low-mass stars below $2.0 M_{\odot}$ in which a helium-flash occurs, we do not include table points between evolutionary points number 6 and 7 . 7 http://obswww . unige.ch/Recherche/evol/-Database-
}

our models are similar, apart from the larger number of species considered by STAREVOL. STAREVOL has indeed a more extended network of nuclear reactions than the Geneva code, which allows us to follow in particular the evolution of unstable elements such as ${ }^{13} \mathrm{~N},{ }^{15} \mathrm{O}$, and ${ }^{7} \mathrm{Be}$. As a consequence, the convective cores are smaller on the main sequence in STAREVOL models (by $\sim 7 \%$ for the $\left[4.0 M_{\odot}, Z_{\odot}\right]$ model), and the tracks in the HR-diagram are slightly less luminous $\left(L_{\mathrm{TO}}\right.$ is $\sim 10 \%$ lower for the $\left[4.0 M_{\odot}, Z_{\odot}\right]$ model). This results in a difference in the lifetime on the main sequence of $4 \%$ for the $\left[4.0 M_{\odot}, Z_{\odot}\right]$ model. This is the main difference we could identify when comparing standard models computed with the two codes.

For the rotational transport, both codes follow the advection by meridional circulation and the diffusion by shear turbulence. We carefully checked that the use of a different prescription for the turbulent diffusion coefficient $D_{v}$ (Talon \& Zahn 1997, in STAREVOL; and Maeder 1997, in Geneva code) has no impact in the mass domain we explore, as the mixing above the convective core is dominated by $D_{\text {eff. }}$. Both codes use prescriptions from Zahn (1992) for the horizontal diffusion coefficient, $D_{\mathrm{h}}$. However, in STAREVOL we always use Eq. (7) that is given by Zahn (1992) to avoid the divergence in the effective diffusion coefficient $\left(\propto \frac{1}{D_{\mathrm{h}}}\right)$, while in the Geneva code the original Eq. (2.29) of Zahn (1992) is used. Surprisingly, Eq. (7) implies higher values of $D_{\mathrm{h}}$ at the edge of the convective core (by about a factor of four in the $\left[4.0 M_{\odot}, Z_{\odot}\right]$ model on the main sequence), which leads to less mixing in these central regions. Combined with the STAREVOL models having a lower initial rotation velocity on the ZAMS, this results in a shorter lifetime and lower luminosity (the maximum difference being $15 \%$ for the $\left[4.0 M_{\odot}\right.$, $\left.Z_{\odot}\right]$ model).

\section{Global properties of the models}

The effects of rotation-induced mixing and thermohaline instability on the surface chemical properties of the grid stars were extensively discussed in Papers I and II. Here we focus on the global properties of the models.

\subsection{Hertzsprung-Russell diagrams and $\log T_{c}$ versus $\log \rho_{c}$}

The evolutionary tracks in the HR diagram are shown in Fig. 2 for all the models computed in this study, with and without rotation. We also present the evolution of the central temperature and density in Fig. 3 for the solar-metallicity case.

\subsubsection{Metallicity effects}

We first recall the impact of metallicity as already reported in the literature (e.g. Schaller et al. 1992; Schaerer et al. 1993; Charbonnel 1994; Heger \& Langer 2000; Maeder 2009). For a given stellar mass, one notes the following effects when metallicity decreases, owing to a decrease in the radiative opacity:

- the ZAMS is shifted to the blue, and both the stellar luminosity and effective temperature are higher at that phase than for solar metallicity models;

- the positions of the RGB and the AGB are shifted to the blue, and the ignition of helium (in degenerate conditions or not) occurs at a lower luminosity at the tip of the RGB $\left(L=21308 L_{\odot}\right.$ and $21252 L_{\odot}$ for models [1.5 $M_{\odot}, Z=0.014$ and $Z=0.002$ ], respectively); 
Table 2. Description of table containing the results of our evolutionary models, both in the standard case and when including rotation and thermohaline mixing.

\begin{tabular}{|c|c|c|}
\hline Stellar parameters & Surface abundances & Central abundances \\
\hline $\begin{array}{l}\text { - Model number } \\
\text { - Maximum of temperature } T_{\max }(\mathrm{K}) \\
\text { - Mass coordinate of } T_{\max }\left(M_{\odot}\right) \\
\text { - Effective temperature } T_{\mathrm{eff}}(\mathrm{K}) \\
\text { - Surface luminosity } L\left(L_{\odot}\right) \\
\text { - Photospheric radius radius } R_{\mathrm{eff}}\left(R_{\odot}\right) \\
\text { - Photospheric density } \rho_{\mathrm{eff}}\left(\mathrm{g} \mathrm{cm}^{-3}\right) \\
\text { - Density at the location of } T_{\max }, \rho_{\max }\left(\mathrm{g} \mathrm{cm}^{-3}\right) \\
\text { - Stellar mass } M\left(M_{\odot}\right) \\
\text { - Mass-loss rate }\left(M_{\odot} \mathrm{yr}^{-1}\right) \\
\text { - Age } t(\mathrm{yr}) \\
\text { - Photospheric gravity } \log \left(g_{\mathrm{eff}}\right)(\log (\mathrm{cgs})) \\
\text { - Central temperature } T_{\mathrm{c}}(\mathrm{K}) \\
\text { - Central pressure } P_{\mathrm{c}} \\
\text { - Surface velocity } v_{\text {surf }}\left(\mathrm{km} \mathrm{s}^{-1}\right)\end{array}$ & $\begin{array}{c}{ }^{1} \mathrm{H}^{2} \mathrm{H} \\
{ }^{3} \mathrm{He}{ }^{4} \mathrm{He} \\
{ }^{6} \mathrm{Li}{ }^{7} \mathrm{Li} \\
{ }^{7} \mathrm{Be}{ }^{9} \mathrm{Be} \\
{ }^{10} \mathrm{~B}{ }^{11} \mathrm{~B} \\
{ }^{12} \mathrm{C}^{13} \mathrm{C}^{14} \mathrm{C} \\
{ }^{14} \mathrm{~N}^{15} \mathrm{~N} \\
{ }^{16} \mathrm{O}^{17} \mathrm{O}^{18} \mathrm{O} \\
{ }^{19} \mathrm{~F} \\
{ }^{20} \mathrm{Ne}^{21} \mathrm{Ne} \\
{ }^{23} \mathrm{Na} \\
{ }^{24} \mathrm{Mg}^{25} \mathrm{Mg} \\
{ }^{26} \mathrm{Al} \\
{ }^{28} \mathrm{Mg} \\
{ }^{28} \mathrm{Si}\end{array}$ & $\begin{array}{c}{ }^{12} \mathrm{C}^{13} \mathrm{C}^{14} \mathrm{C} \\
{ }^{14} \mathrm{~N} \\
{ }^{16} \mathrm{O}^{17} \mathrm{O}^{18} \mathrm{O} \\
{ }^{19} \mathrm{~F} \\
{ }^{20} \mathrm{Ne}^{21} \mathrm{Ne}^{22} \mathrm{Ne} \\
{ }^{23} \mathrm{Na} \\
{ }^{24} \mathrm{Mg}^{26} \mathrm{Mg}{ }^{26} \mathrm{Mg} \\
{ }^{28} \mathrm{Si}\end{array}$ \\
\hline $\begin{array}{l}\text { - Mass at the base of convective envelope }\left(M_{\odot}\right) \\
\text { - The large separation from scaling relation } \Delta v_{\text {scale }}(v \mathrm{~Hz}) \\
\text { - The large separation from asymptotic relation } \Delta v_{\mathrm{asymp}}(\nu \mathrm{Hz}) \\
\text { - The frequency with the maximum amplitude } v_{\max } \\
\text { - The maximum amplitude } A_{\max } \\
\text { - The asymptotic period spacing of g-modes } \Delta \Pi(\mathrm{s}) \\
\text { - The total acoustic radius } T(\mathrm{~s}) \\
\text { - The acoustic radius at the base of the convective envelope } t_{\mathrm{BCE}}(\mathrm{s}) \\
\text { - The acoustic radius at the location of helium second-ionisation region } t_{\mathrm{He}}(\mathrm{s})\end{array}$ & & \\
\hline
\end{tabular}

- central helium-burning occurs at a higher effective temperature, and more extended blue loops are obtained.

\subsubsection{Impact of rotation-induced mixing and thermohaline instability}

- As has been known for a long time (see e.g. Maeder \& Meynet 2000) and shown in Fig. 2, rotation-induced mixing affects the evolution tracks in the HR diagram. On the main sequence, rotational mixing brings fresh hydrogen fuel into the longer-lasting convective core, and transports H-burning products outwards. This results in more massive helium cores at the turnoff than in the standard case and shifts the tracks toward higher effective temperatures and luminosities throughout their evolution (Ekström et al. 2012; Heger \& Langer 2000; Maeder \& Meynet 2000; Meynet \& Maeder 2000). The right panel of Fig. 3 shows the central conditions in standard and rotating models for selected masses at solar metallicity. Rotating models behave as stars with higher masses throughout their evolution.

- Thermohaline mixing induced by ${ }^{3} \mathrm{He}$-burning becomes efficient only on the RGB at the bump luminosity (Charbonnel \& Zahn 2007b; Charbonnel \& Lagarde 2010). Beyond this point, the double-diffusive instability develops in a very thin region located between the hydrogen-burning shell and the convective envelope, and has a negligible effect on the stellar structure. It does not modify the evolutionary tracks in the HR diagram nor the $\log T_{\mathrm{c}}$ versus $\log \rho_{\mathrm{C}}$ diagram.

\subsection{Lifetimes}

The theoretical lifetimes are shown in Fig. 4 for the main phases of evolution as a function of the initial stellar mass and for the four metallicities considered, with the effects of rotation being clearly illustrated. We recall the main points:

- The duration of all the evolutionary phases decreases when the initial stellar mass increases and when the metallicity decreases (Schaller et al. 1992; Schaerer et al. 1993; Charbonnel 1994), which is consistent with the abovementioned luminosity and effective-temperature differences.

- After the bump luminosity, the thermohaline mixing does not affect the lifetimes on the RGB and on the early-AGB phases.

- The lifetimes of rotating models on the main sequence are longer than those of the standard models. Indeed rotationinduced mixing brings fresh hydrogen fuel into the stellar core during that phase. As a consequence, the exhaustion of hydrogen in the central region is delayed and the lifetime on the main sequence lengthens; in addition, the mass of the helium-core is larger at the end of the main sequence when rotation is accounted for (Ekström et al. 2012; Heger \& Langer 2000; Maeder \& Meynet 2000; Meynet \& Maeder 2000).

- As a consequence of having a more massive core at the end of the main sequence due to rotation, the models that undergo the helium-flash spend a shorter time on the RGB (see Fig. 4). As for the Main Sequence (MS), all rotating models have a longer lifetime during the quiescent central He burning phase.

- Lifetimes on the early-AGB are longer in rotating models than in standard models, owing to their more massive core. This remains unchanged if we add the lifetime on the TP-AGB $\left(1.1 \times 10^{6} \mathrm{yr}\right.$ for $\left[1.5 M_{\odot}, Z_{\odot}\right]$ and $2 \times 10^{6} \mathrm{yr}$ for $\left[3 M_{\odot}, Z_{\odot}\right]$ ) to the early-AGB, as the total lifetime on the AGB is only increased by a few percent. 
N. Lagarde et al.: Grid of stellar models and asteroseismic quantities

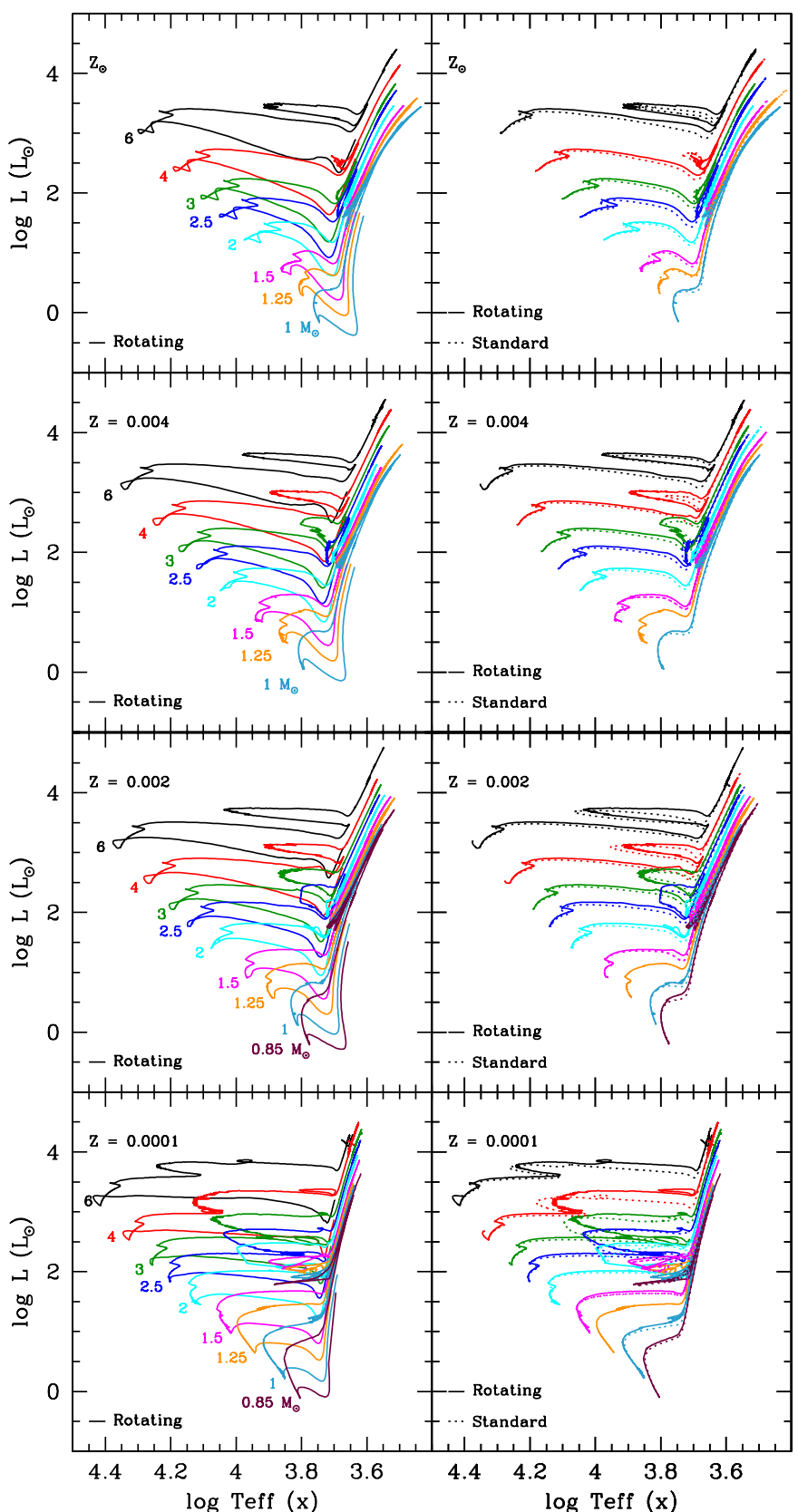

Fig. 2. Theoretical evolution tracks in the HR diagram for the ensemble of calculated models for all metallicities $\left(Z_{\odot}, Z=0.004, Z=0.002\right.$, and $Z=0.0001$, from top to bottom). In the left panels, the tracks are shown for the rotating models from the pre-main sequence on. In the right panels, both standard and rotating predictions are shown (dashed and solid lines respectively) from the ZAMS and beyond.

- As seen in previous subsections, thermohaline mixing has a negligible impact on the stellar structure, and modifies neither the stellar evolutionary tracks nor lifetimes.

\section{Global asteroseismic quantities}

A large number of asteroseismic observations have been obtained for different kinds of stars. In particular, the detection and characterization of solar-like oscillations in a large number of red giants by space missions (e.g. De Ridder et al. 2009) promises to provide valuable and independent constraints of current stellar models. The comparison of models including a detailed description of transport processes in stellar interiors and these asteroseismic constraints promises to help us improve our understanding of stars.

Rotation is one of a number of key processes that change all outputs of stellar models (see Sect. 4) with a significant impact on asteroseismic observables. In the case of main-sequence solar-type stars, rotation is found to shift the evolutionary tracks to the blue part of the HR diagram resulting in higher values of the large frequency separation for rotating models than for nonrotating ones at a given evolutionary stage (Eggenberger et al. 2010a). For red giants, rotating models are found to decrease the determined value of the stellar mass of a star located at a given luminosity in the HR diagram and to increase the value of its age. Consequently, the inclusion of rotation significantly changes the fundamental parameters determined for a star by performing an asteroseismic calibration (Eggenberger et al. 2010b).

\subsection{Scaling relations and asymptotic quantities provided for the present grid}

For this new grid of standard and rotating models, we now provide the values of different asteroseismic parameters (see Table 2) that can be directly computed from global stellar properties using scaling relations. We also use the information on the internal structure of models to compute asymptotic relations. These scaling relations are particularly useful for constraining stellar parameters and obtaining additional information about stellar evolution without the need to perform a full asteroseismic analysis (see e.g. Stello et al. 2009; Miglio et al. 2009; Hekker et al. 2009; Mosser et al. 2010; Chaplin et al. 2011b; Beck et al. 2011; Bedding et al. 2011). Although solar-like oscillations are expected to be excited in relatively cool stars (main sequence, as well as red giant stars), these global asteroseismic quantities are provided for all models of our grid and not only for models with a significant convective envelope.

The first global asteroseismic quantity we provide is the large frequency separation $\Delta v$, which is expected to be proportional to the square root of the mean stellar density (e.g. Ulrich 1986)

$\Delta v_{\text {scale }}=\Delta v_{\odot}\left(\frac{M}{M_{\odot}}\right)^{0.5}\left(\frac{R}{R_{\odot}}\right)^{-1.5}$,

with the solar large-frequency separation $\Delta v_{\odot}=134.9 \mu \mathrm{Hz}$.

Belkacem et al. (2011) proved that the frequency $v_{\max }$ at which the oscillation modes reach their strongest amplitudes is approximatively proportional to the acoustic cut-off frequency, as suspected by Brown et al. (1991) and Kjeldsen \& Bedding (1995), such that

$v_{\text {max }}=v_{\text {max }, \odot}\left(\frac{M}{M_{\odot}}\right)\left(\frac{R}{R_{\odot}}\right)^{-2}\left(\frac{T_{\mathrm{eff}}}{T_{\mathrm{eff}, \odot}}\right)^{-0.5}$,

for the solar value $v_{\max , \odot}=3150 \mu \mathrm{Hz}$.

Finally, the value of maximum oscillation amplitude relative to that of the Sun $\left(A_{\max } / A_{\max , \odot}\right)$ is computed using the relation (e.g. Huber et al. 2011)

$A_{\max } / A_{\max , \odot}=\frac{\left(L / L_{\odot}\right)^{s}}{\left(M / M_{\odot}\right)^{t}}\left(\frac{T_{\mathrm{eff}}}{T_{\mathrm{eff}, \odot}}\right)^{-r}$,

for the solar value from Kepler $A_{\max , \odot}=2.5 \mathrm{ppm}$.

Following Huber and collaborators, we adopt $s=0.838$ and $t=1.32$. A value of $r=2$ is adopted as advocated by Kjeldsen $\&$ Bedding (1995). We note that we simply assume that these relations hold for the different models computed here and that we do 

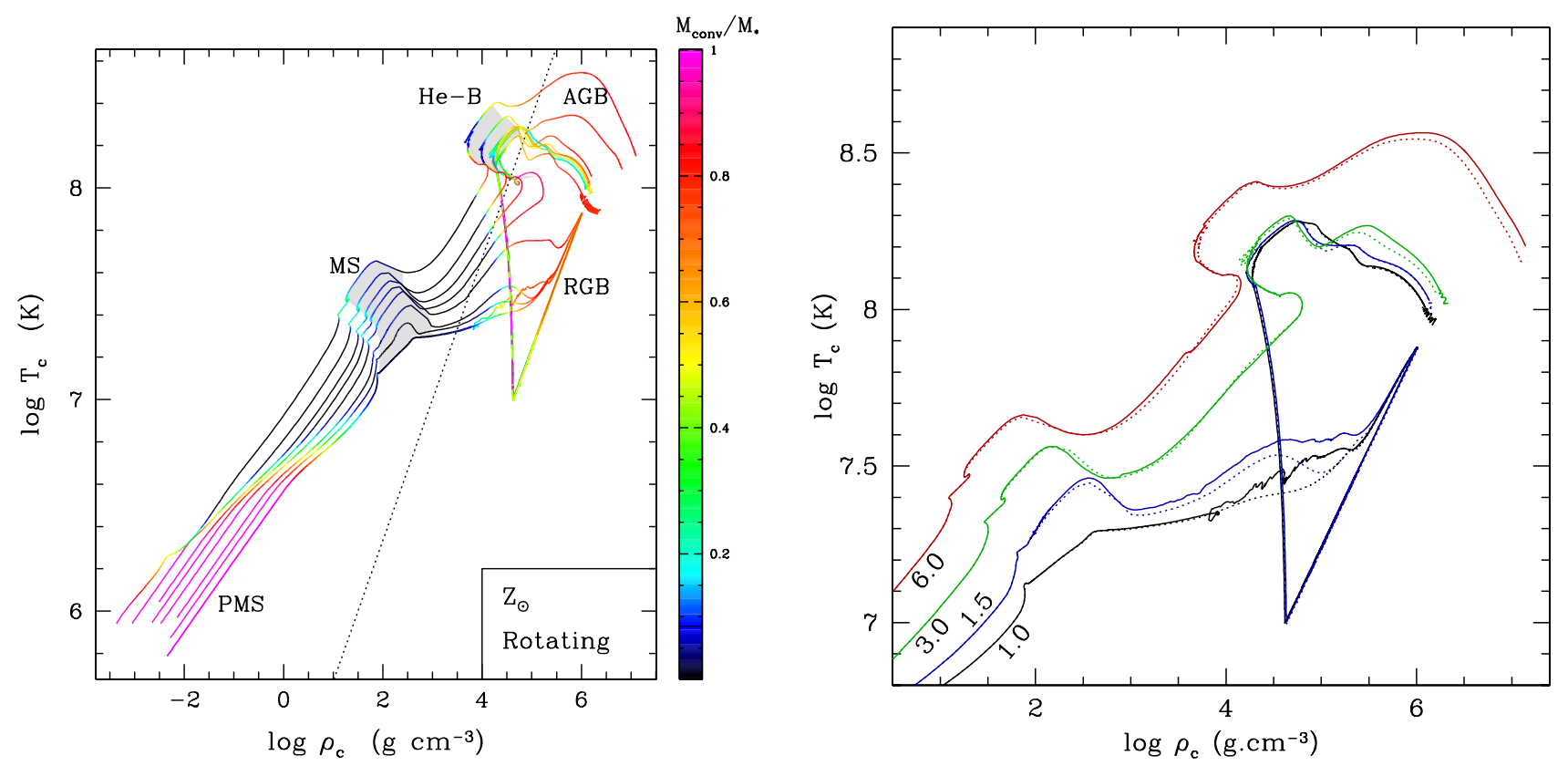

Fig. 3. Left panel: central density as a function of central temperature for the rotating $Z_{\odot}$ models. Colours indicate the mass of convective regions (convective core and convective envelope) over the total stellar mass. Shaded regions indicate the phases of central hydrogen-burning (MS) and helium-burning (He-B). Right panel: central density as a function of central temperature for standard and rotating models (dashed and solid lines respectively) for four initial stellar masses from the ZAMS beyond.
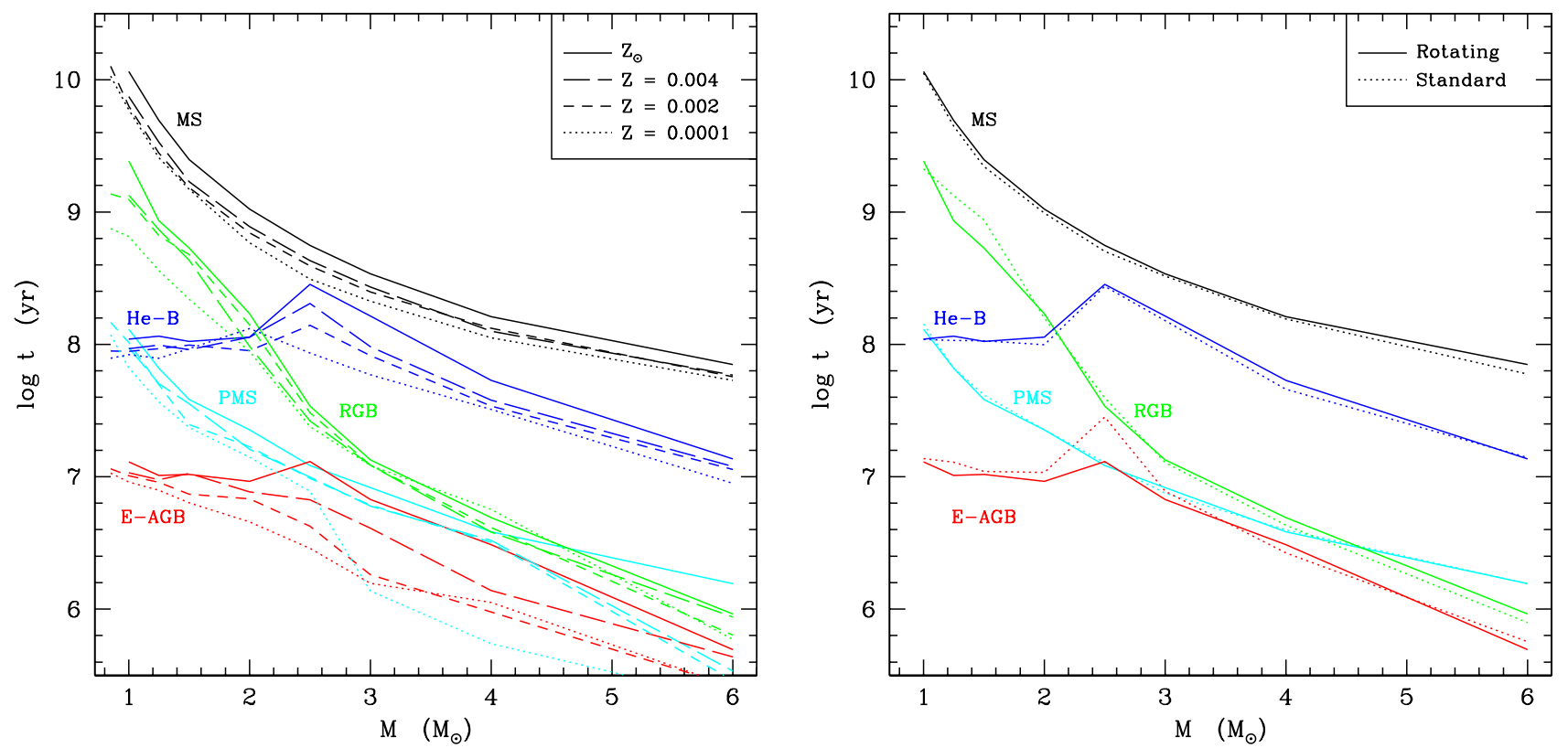

Fig. 4. Duration of the different evolution phases as a function of the initial stellar mass. (Left) Effects of metallicity in the standard models. (Right) Effects of rotation at solar metallicity.

not test the validity of the scaling relations. Preliminary studies suggest that these simple scaling relations hold reasonably well for main sequence and RGB stars (e.g. Stello et al. 2009; White et al. 2011).

In addition to the asteroseismic observables deduced from scaling relations, asymptotic asteroseismic quantities are also provided.

The asymptotic large frequency separation is given by

$\Delta v_{\text {asymp }}=\left(2 \int_{0}^{R} \frac{\mathrm{d} r}{c_{\mathrm{s}}}\right)^{-1}$,

where $R$ is the stellar radius and $c_{\mathrm{s}}$ is the sound speed.
The total acoustic radius $T$ is directly related to the large frequency separation, such that

$T=\int_{0}^{R} \frac{\mathrm{d} r}{c_{\mathrm{s}}}=\frac{1}{2 \cdot \Delta \nu_{\mathrm{asymp}}}$.

The acoustic radius at the base of convective envelope $\left(t_{\mathrm{BCE}}\right)$ and the location of the helium second-ionisation region ${ }^{8}\left(t_{\mathrm{He}}\right)$

8 The Schwarzschild citerion allows us to define the base of the convective envelope. The mininum in $\Gamma_{1}$, the adiabatic exponent, corresponding to He-II defines the location of the helium second-ionisation region. 

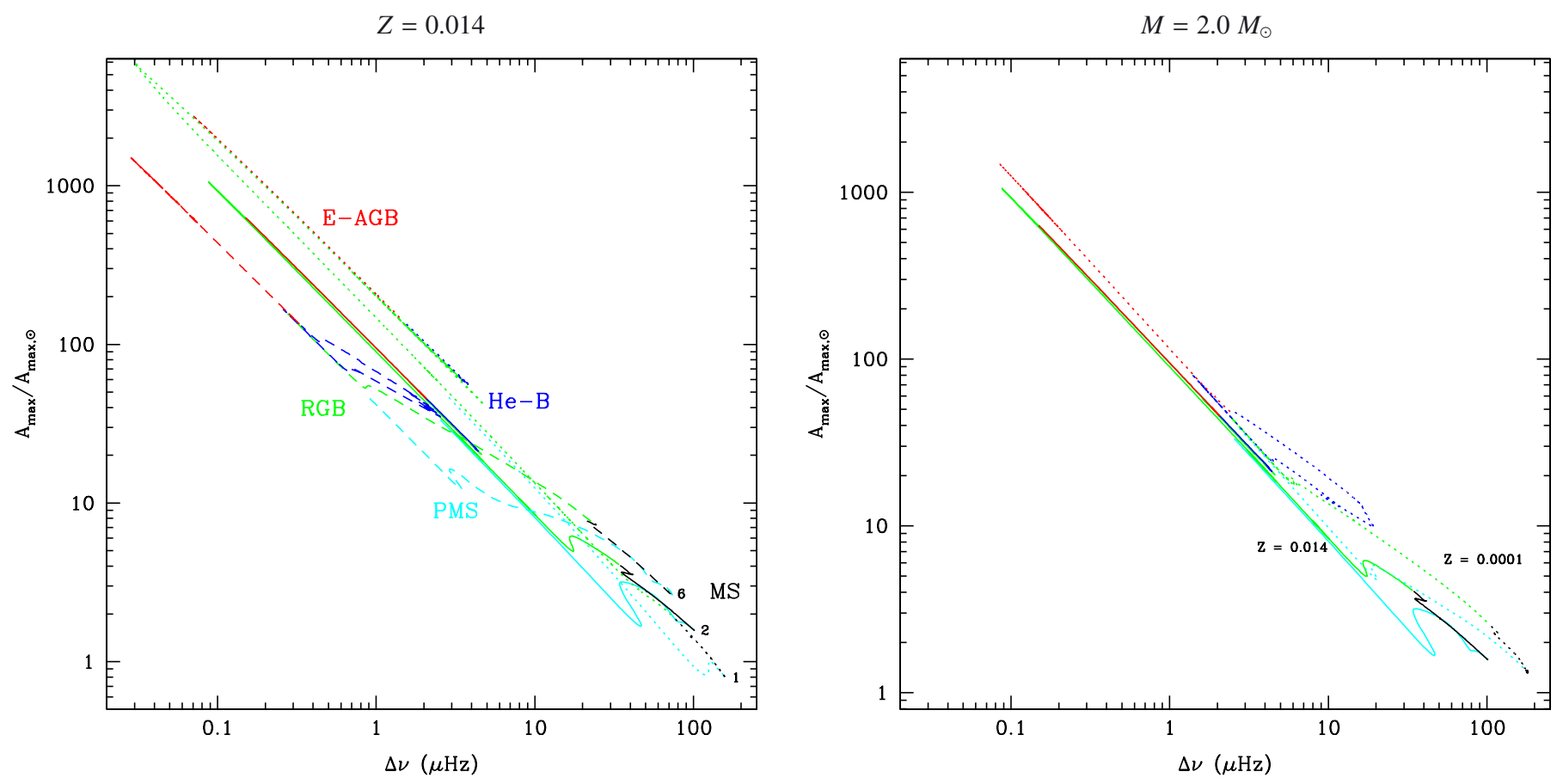

Fig. 5. Maximal amplitude compared to solar as a function of the large separation: (left panel) at solar metallicity for $1.25 M_{\odot}, 2.0 M_{\odot}$, and $6.0 M_{\odot}$ rotating models, and (right panel) at two metallicities $Z=0.014$ (solid lines) and $Z=0.0001$ (dotted-lines) for a $2.0 M_{\odot}$ rotating model. Evolutionary phases are color-labeled: pre-main sequence (cyan), main sequence (black), RGB (green), helium-burning (blue), and AGB (red).

are determined with the relations

$t_{\mathrm{BCE}}=\int_{0}^{r_{\mathrm{BCE}}} \frac{\mathrm{d} r}{c_{\mathrm{S}}}, t_{\mathrm{He}}=\int_{0}^{r_{\mathrm{He}}} \frac{\mathrm{d} r}{c_{\mathrm{S}}}$,

where $r_{\mathrm{BCE}}$ and $r_{\mathrm{He}}$ represent the stellar radius at the base of convective envelope and the location of the helium secondionization zone, respectively.

The period spacing of gravity modes for $\ell=1$ and different acoustic radii can be determined with the asymptotic relation

$\Delta \Pi(\ell=1)=\frac{2^{\frac{1}{2}} \cdot \pi^{2}}{\int_{r_{1}}^{r_{2}} N \cdot \frac{\mathrm{d} r}{r}}$,

where $N$ is the Brunt-Väisälä frequency, and $r_{1}$ and $r_{2}$ define the domain (in radius) where the $\mathrm{g}$ modes are trapped. Within this region, the mode frequency $(\omega)$ must satisfy the conditions

$\omega^{2}<N^{2}$

and

$\omega^{2}<S_{1}^{2}=\frac{l(l+1) c_{\mathrm{s}}^{2}}{r^{2}}$

where $S_{1}$ is the Lamb frequency.

In the following discussions, we use the large separation determined with the scaling relation $\Delta v_{\text {scale }}$ (noted $\Delta v$ in the following sections). We have compared the large separation estimates $\Delta v_{\text {scale }}$ (Eq. (8)) and $\Delta v_{\text {asymp }}$ (Eq. (11)) to evaluate the difference between both expressions. On the main sequence, this difference varies between $3 \%$ and $5 \%$ for a $1.0 M_{\odot}$ model at solar metallicity. We find that the relative error obtained when using either Eqs. (8) or (11) depends on the stellar mass (i.e. 3-8\% for $\left[2.0 M_{\odot}, Z_{\odot}\right] \mathrm{MS}$ model), the metallicity (i.e. $10-12 \%$ for the [2.0 $\left.M_{\odot}, Z=10^{-4}\right] \mathrm{MS}$ model), and the evolutionary phase (i.e. $<15 \%$ on RGB, $<10 \%$ on He-B both for $\left[1.0 M_{\odot}, Z_{\odot}\right]$ model). In a future paper, we propose to interpret these differences and investigate how they compare to White et al. (2011).

\subsection{Evolution of the asteroseismic observables}

We now describe the global asteroseismic properties of lowand intermediate-mass stars during their evolution, and discuss the effects of varying the stellar mass, metallicity, and the nonstandard mixing mechanisms. We illustrate our discussion in Figs. 5 to 10. In Fig. 5, we present the maximal oscillation amplitude as a function of the large frequency separation throughout the stellar evolution from the pre-main sequence to the AGB phase for three different initial stellar masses at solar metallicity (left panel) and for a $2 M_{\odot}$ star between the lowest $(Z=0.0001)$ and the highest $(Z=0.014)$ metallicities explored here (right panel). The line colors change with the stellar evolution phases: cyan corresponds to the pre-main sequence, black to the main sequence, green to the red giant branch, blue to core heliumburning, and red to the AGB. Figure 6 presents the same quantities for all the stellar masses considered at solar metallicity, for both the standard and the rotating cases (dashed and solid lines, respectively), each evolutionary phase being presented singly.

\subsubsection{Trends for a given stellar mass and metallicity - the case of the $2.0 M_{\odot}, Z_{\odot}$ models}

We now describe the evolution of the seismic properties of a star of $2.0 M_{\odot}$ at solar metallicity as depicted by the solid line in Fig. 5 and the black line in Fig. 6. Although this $2 M_{\odot}$ star is not expected to undergo solar-like oscillations during the main sequence owing to its too high surface temperature, it represents a typical model of a red giant exhibiting solar-like oscillations. Moreover, the discussion in the changes of the asteroseismic quantities during the main sequence remains globally valid for less-massive main-sequence stars (the main difference being the value of the ratio of the acoustic radius at the base of the convective envelope to the total acoustic radius, see Fig. 8). 
Pre-main sequence

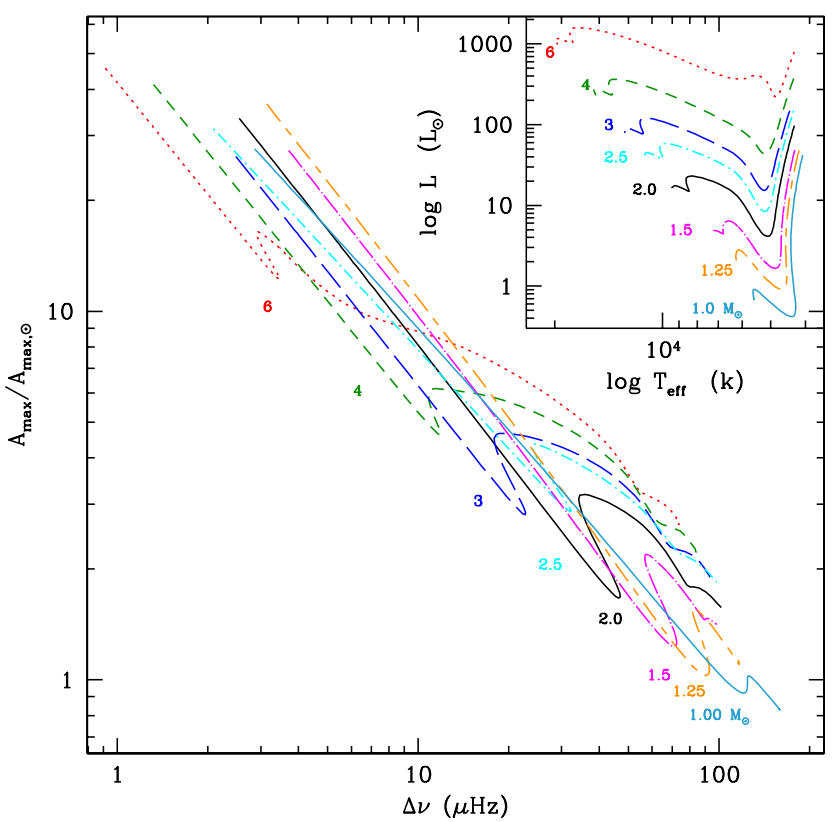

Red giant branch

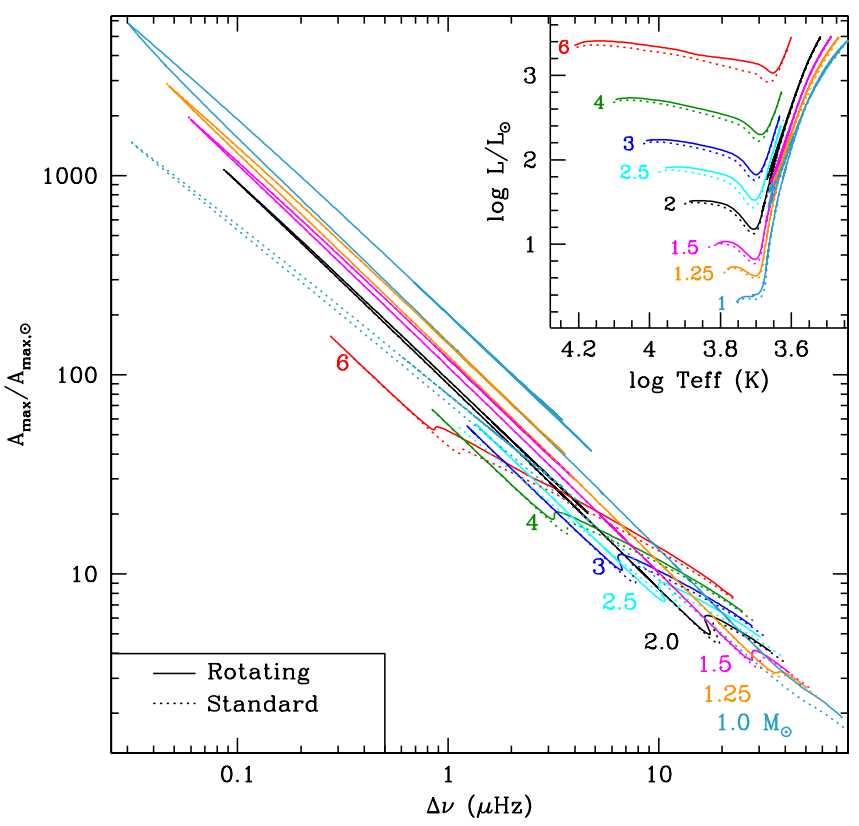

Main sequence

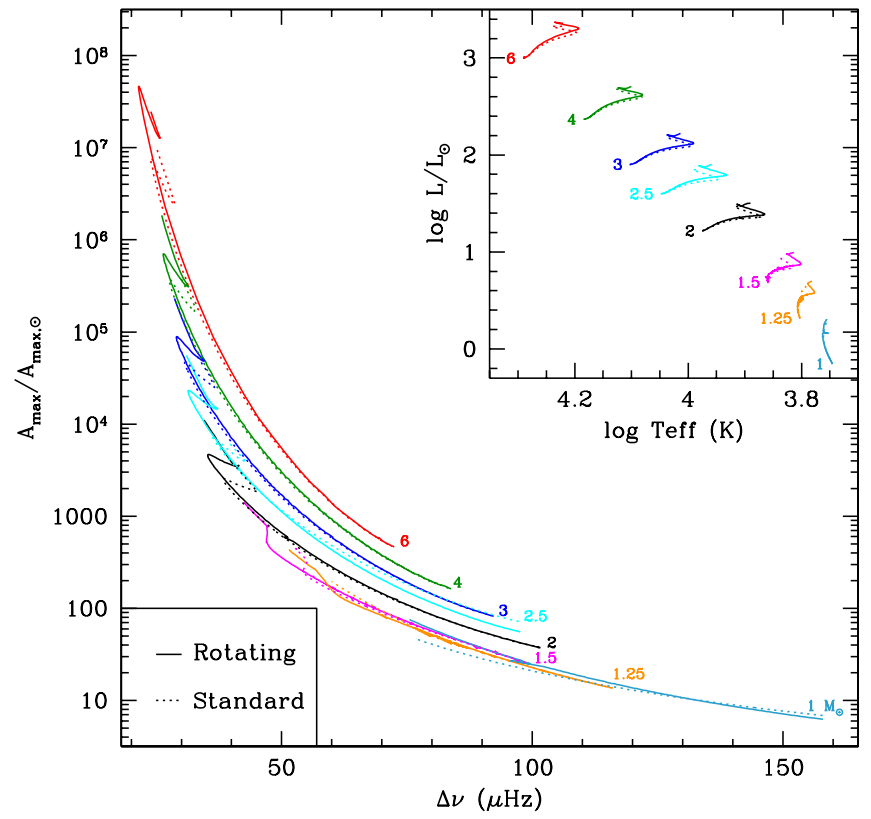

Helium-burning

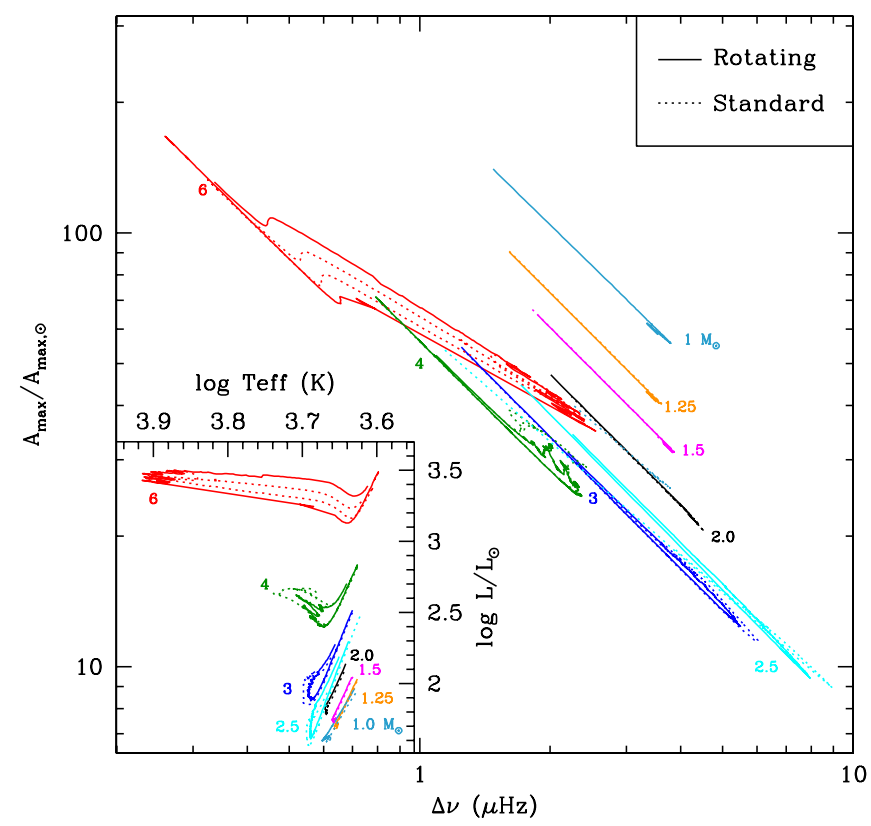

Fig. 6. Maximal amplitude compared to solar as a function of the larger separation for models at $Z_{\odot}$. Top-left panel: on the pre-main sequence; topright panel: on the main sequence; bottom-left panel: on the red giant branch; bottom-right panel: on helium-burning phase. Standard models and rotating models are shown with dashed and solid lines respectively except for pre-main sequence. The corresponding evolution in the HertzsprungRussell diagram is shown on the right corner of each panel.

The large separation $\Delta v$ increases along the pre-main sequence because of its inverse dependence on stellar radius, which decreases during that phase (see Eq. (14)). On the other hand, the simultaneous decrease in maximal amplitude $A_{\max } / A_{\max , \odot}$ results from the decrease in luminosity and from the rise in effective temperature (see Eq. (10)).

On the main sequence, $A_{\max } / A_{\max , \odot}$ rises because of its dependence on both luminosity and the inverse of the effective temperature. In addition, the expansion of the stellar radius causes $\Delta v$ to increase. The radius increase also causes the large separation to drop significantly as the model evolves on the RGB, while the maximum amplitude increases during this phase as a result of the luminosity increase and the effective temperature decrease.

After the helium-flash at the tip of the RGB, the luminosity and the stellar radius dwindle with a rise in effective temperature, implying that the maximum of the amplitude wanes as the large separation increases. Throughout the helium-burning 


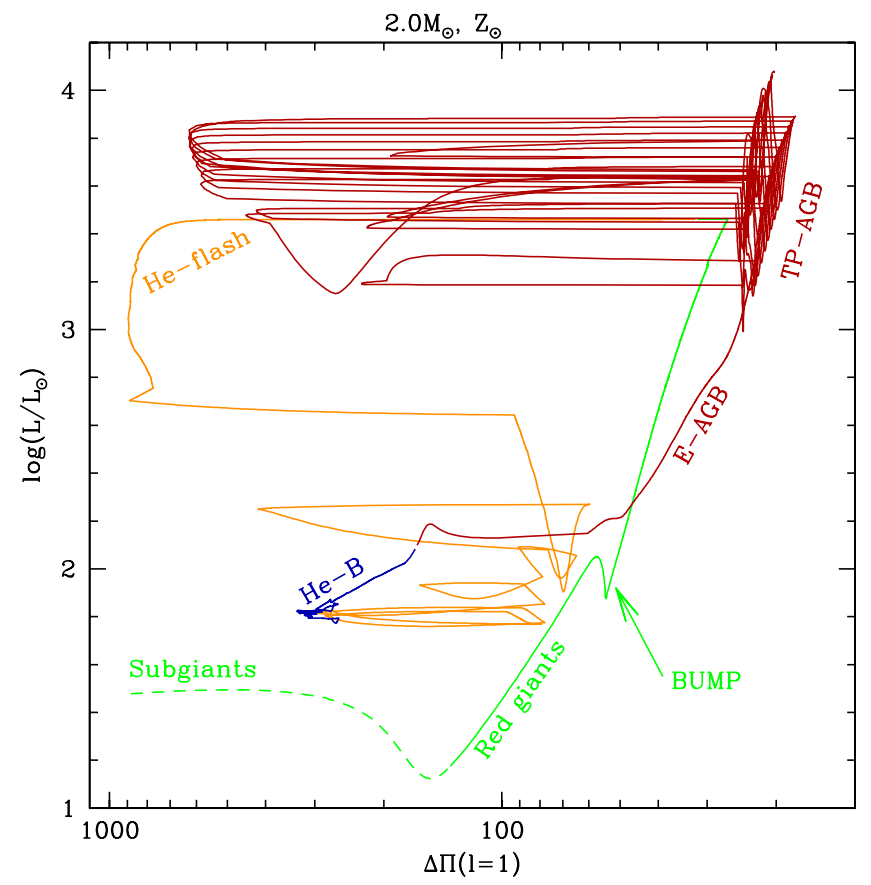

Fig. 7. The stellar luminosity as a function of the asymptotic period spacing of g-modes for the standard $2.0 M_{\odot}$ model at solar metallicity. Evolutionary phases are color-labeled: subgiant (green dashed), red giant (green solid), helium-flash episode (orange), helium-burning (blue), and asymptotic giant branch (red).

phase, the luminosity and stellar radius increase, while the effective temperature and stellar mass decrease. Consequently, a gain in $A_{\max }$ is obtained, while the large separation reduces.

Figure 7 presents the asymptotic period spacing of gravity modes $\Delta \Pi(\ell=1)$ for the standard model of $2.0 M_{\odot}$ at solar metallicity. As proposed by Bedding et al. (2011) and Mosser et al. (2011), this quantity allows us to distinguish two stars that have the same luminosity, one being at the RGB bump and the other one being at the clump undergoing central He burning. At $\log \left(L / L_{\odot}\right) \sim 2.0$, we indeed find that $\Delta \Pi(\ell=1)=55 \mathrm{~s}$ at the RGB bump, and $\Delta \Pi(\ell=1)=190 \mathrm{~s}$ in the clump. As the stellar structure, and more particularly the presence of the convective core affects the domain where the g-modes are trapped, $\Delta \Pi(\ell=1)$ is larger in clump stars than in RGB stars (Christensen-Dalsgaard 2011). Large variations in $\Delta \Pi(\ell=1)$ are observed during the thermal pulses and helium-flash phase because of the formation of an intermediate convective zone during the helium-flashes and thermal pulses. We assume that the modes are trapped in the outermost radiative zone leading to a large value of $\Delta \Pi(\ell=1)$ (Bildsten et al. 2012).

Figure 8 shows the acoustic radius at the bases of convective envelope $\left(t_{\mathrm{BCE}}\right)$ and helium-ionization zone $\left(t_{\mathrm{He}}\right)$, both over the total acoustic radius as a function of luminosity, for the standard (black line) $1.0 M_{\odot}$ (left panel) and $2.0 M_{\odot}$ (right panel) models at solar metallicity from the main sequence to heliumburning phase. As the extent (in radius) of the convective envelope decreases with increasing stellar mass on the main sequence, the difference between $t_{\mathrm{He}}$ and $t_{\mathrm{BCE}}$ becomes smaller.

As the convective envelope deepens inside the star with the first dredge-up, the acoustic radius $t_{\mathrm{BCE}}$ decreases during the subgiant branch. During this phase, the acoustic radius at the base of $\mathrm{He}$ II ionization zone follows the variation in effective temperature and $t_{\mathrm{He}}$ increases while $T_{\text {eff }}$ decreases. In addition, the total acoustic radius increases because of its dependence on the stellar radius, which increases during this phase.
Consequently, $t_{\mathrm{He}} / T$ decreases from 0.8 to 0.68 and from 0.9 to 0.7 for $\left[1.0 M_{\odot}, Z_{\odot}\right]$ and $\left[2.0 M_{\odot}, Z_{\odot}\right]$ models, respectively. As the star ascends the RGB, $t_{\mathrm{BCE}}$ follows the convective envelope and the acoustic radius $t_{\mathrm{He}} / T$ decreases to 0.2 for the $2.0 M_{\odot}$ model until the RGB tip is reached (at $\left.\log L / L_{\odot} \sim 3.5\right)$. When the stellar luminosity decreases after the RGB tip, the convective envelope radius decreases, and the effective temperature increases as the star contracts. Therefore, $t_{\mathrm{BCE}} / T$ and $t_{\mathrm{He}} / T$ increase until the central temperature is sufficient to ignite helium. At the end of the He burning phase, the surface layers expand and the convective envelope deepens as the effective temperature decreases. During this phase, $t_{\mathrm{BCE}} / T$ and $t_{\mathrm{He}} / T$ in $2.0 M_{\odot}$ model decrease slowly from $\sim 0.62$ and $\sim 0.1$ to $\sim 0.55$ and $\sim 0.05$, respectively.

Although we do not include this phase in the files presented in Sect. 3.1, we discuss the evolution of the asteroseismic parameters during the TP-AGB. Figure 10 shows the evolution of the stellar parameters $\left(L, T_{\text {eff }}\right.$, and $\left.M\right)$, and asteroseismic parameters ( $\Delta v, v_{\max }$, and $A_{\max }$ ) as a function of time from the first thermal pulse for a $2.0 M_{\odot}$ model at $Z_{\odot}$.

Between each thermal pulse, the stellar radius increases. The mass slowly decreases by steps at this phase, remaining almost constant during the inter-pulses. The large separation then decreases between each thermal pulse as well as $v_{\max }$ despite the slight decrease in effective temperature. The maximal amplitude increases as a result of the luminosity increase and the decrease in the effective temperature and total stellar mass. The strong increase in $A_{\max }$ during the interpulses is due to the large mass loss at this phase, which can be moreclearly seen in the last pulse shown in Fig. 10.

\subsubsection{Effects of metallicity}

Although the current asteroseismic missions focus on solar metallicity stars, we present the effects of metallicity on the global asteroseismic parameters in Fig. 5 (right panel) for a $2.0 M_{\odot}$ model.

As discussed in Sect. 4.1.1, at lower metallicity the main sequence is shifted to both the blue and higher luminosity on the HR diagram. Therefore, the track in the $A_{\max }$ vs. $\Delta v$ plot is moved to lower $A_{\max } / A_{\max , \odot}$ values. In addition, the luminosity of the helium-ignition at the RGB tip occurs at lower $A_{\max } / A_{\max , \odot}$ when the metallicity is lower. The evolutionary track in the $A_{\max }$ vs. $\Delta v$ plot is shifted to the right (toward higher $\Delta v$ values) when the metallicity decreases. This is a direct consequence of a more metal-poor star behaving as a more massive star.

\subsubsection{Effects of stellar mass}

As the mass increases, the ZAMS (point at higher $\Delta v$ and lower $A_{\max }$ ) is shifted to lower $\Delta v$ and higher $A_{\max }$ (see Fig. 5 and top panels of Fig. 6). Indeed, the large frequency separation at the point of ZAMS decreases because of its dependence on the mean stellar density. As in the case of the Hayashi tracks during the pre-main sequence, stars with different masses tend to join together in the $A_{\max }$ vs. $\Delta v$ plot during the RGB and early-AGB phase (see green and red lines, respectively in Fig. 5). As stellar mass increases, stars follow more extended blue loops during the central helium-burning phase. As shown in Fig. 6 (bottom right panel), blue loops range over high $\Delta v$ and low $A_{\max } / A_{\max , \odot}$.

Figure 9 shows the theoretical evolution of the stellar acoustic radius at the base of the helium-ionization zone and the asymptotic period spacing of g-modes along the evolutionary 

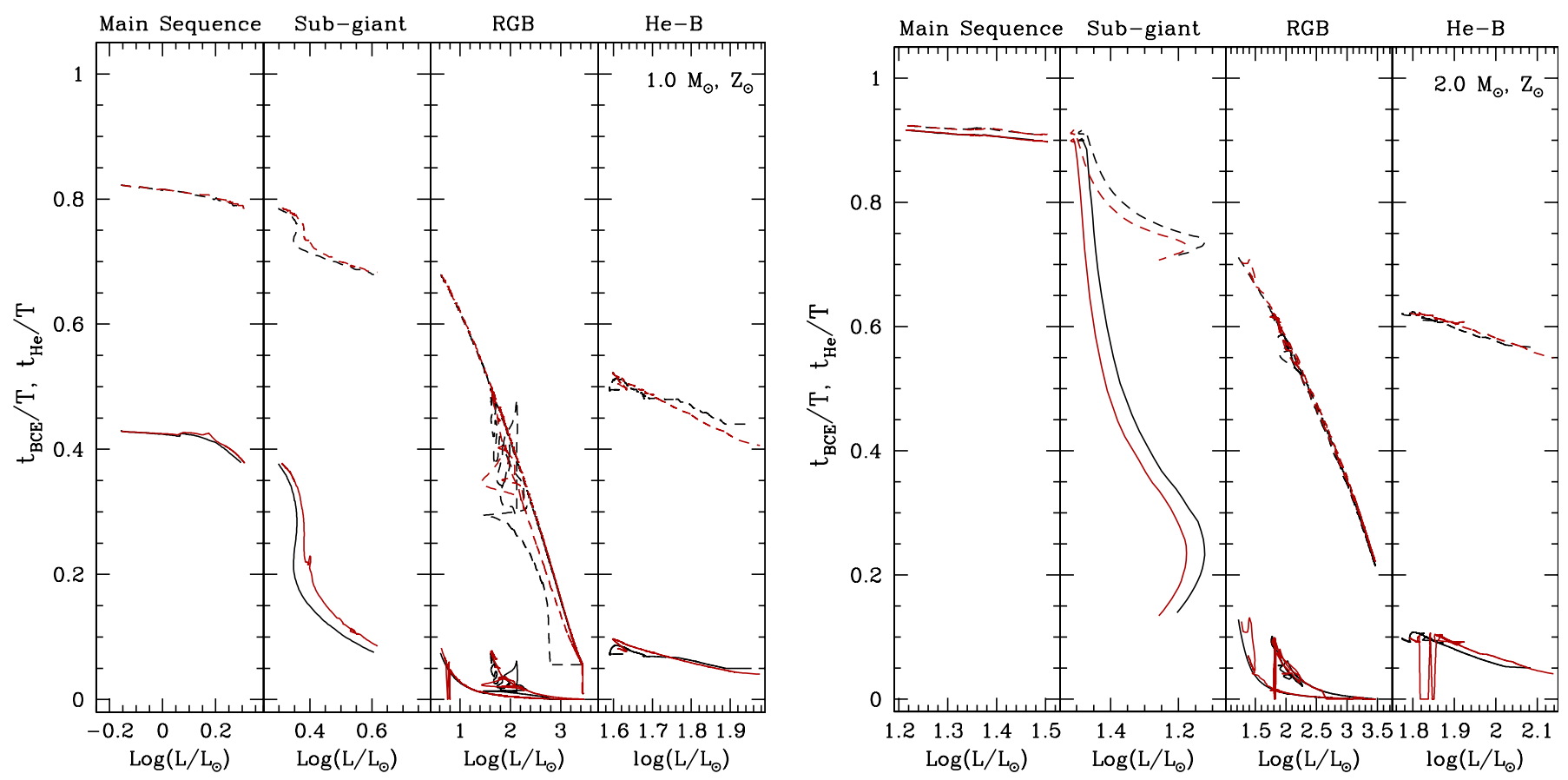

Fig. 8. Acoustic radius at the base of the convective envelope ( $t_{\mathrm{BCE}}$, solid line) and at the base of helium-ionization zone $\left(t_{\mathrm{He}}\right.$, dashed line) compared to total acoustic radius $T$ as a function of the stellar luminosity for (left panel) 1.0 and (right panel) $2.0 M_{\odot}$ models at solar metallicity following standard (black line) and rotating prescriptions (red line); (from left to right) on the main sequence, the sub-giant branch, red giant branch, and helium burning phase.
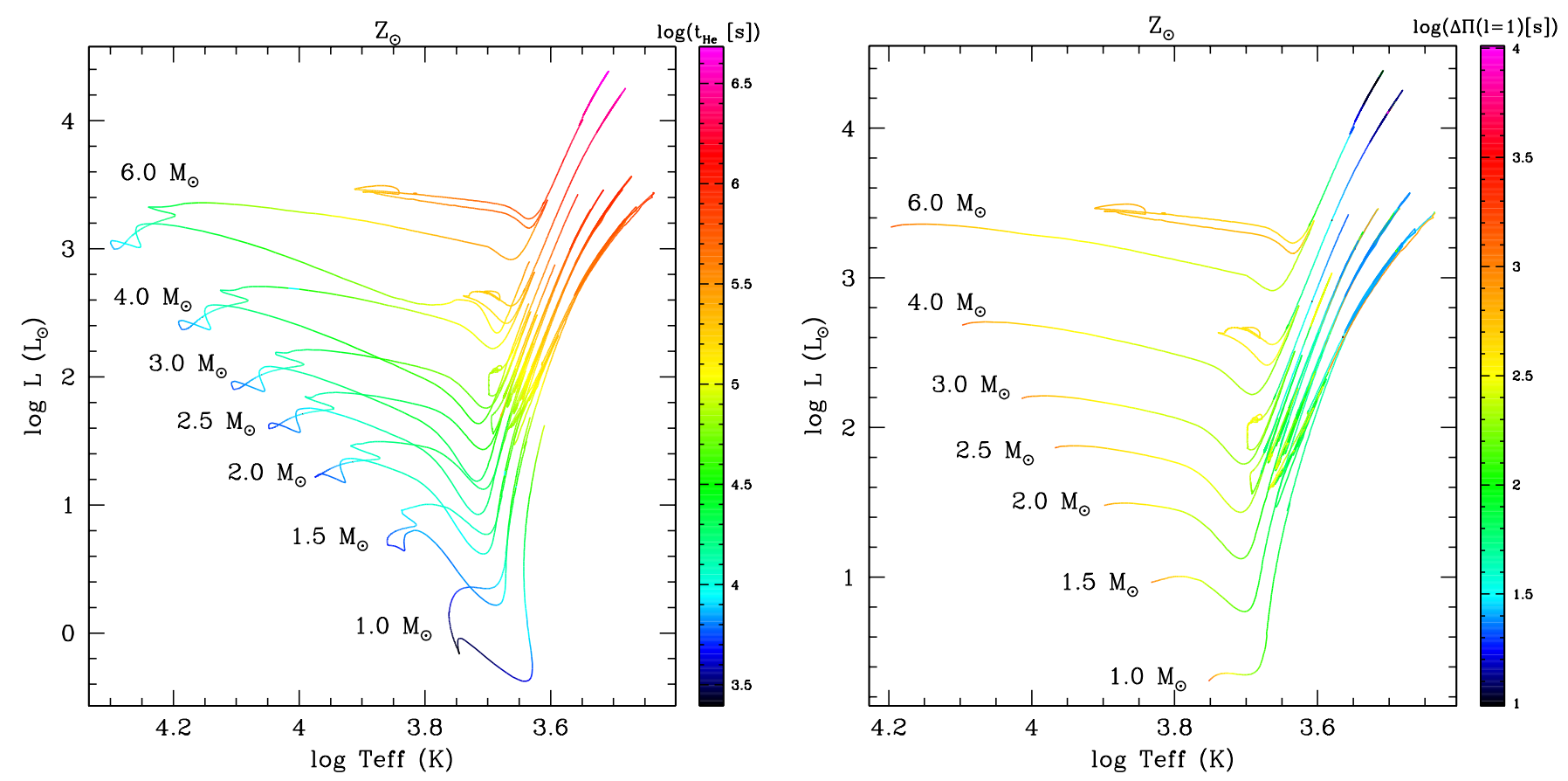

Fig. 9. Color-coded HR diagram for all the masses in the grid at solar metallicity. The color code represents the values (in seconds) of the acoustic radius at the base of the ionization of He II $t_{\mathrm{He}}$ (left panel) and of the asymptotic period spacing of g-modes $\Delta \Pi(\ell=1)($ right panel). The values increase from blue to red in both cases as shown on the scales on the right of the plots.

tracks in the HR diagram for models of different masses at solar metallicity. The variations in both quantities are expressed in seconds. Evolution is shown from the pre-main sequence to the early-AGB phase and from the subgiant branch to the earlyAGB phase for $t_{\mathrm{He}}$ and $\Delta \Pi$, respectively. At a given evolutionary phase, higher stellar mass implies larger acoustic radius at the base of the helium-ionization zone. The same is true for $\Delta \Pi$.

\subsubsection{Impact of rotation on the evolution within the amplitude vs. $\Delta v$ diagram}

We now focus on the effect of accounting for rotational mixing on the global asteroseismic parameters. As thermohaline mixing solely affects the abundance at both the surface and the external layers of HBS pattern during the giant phase, without modifying 
N. Lagarde et al.: Grid of stellar models and asteroseismic quantities

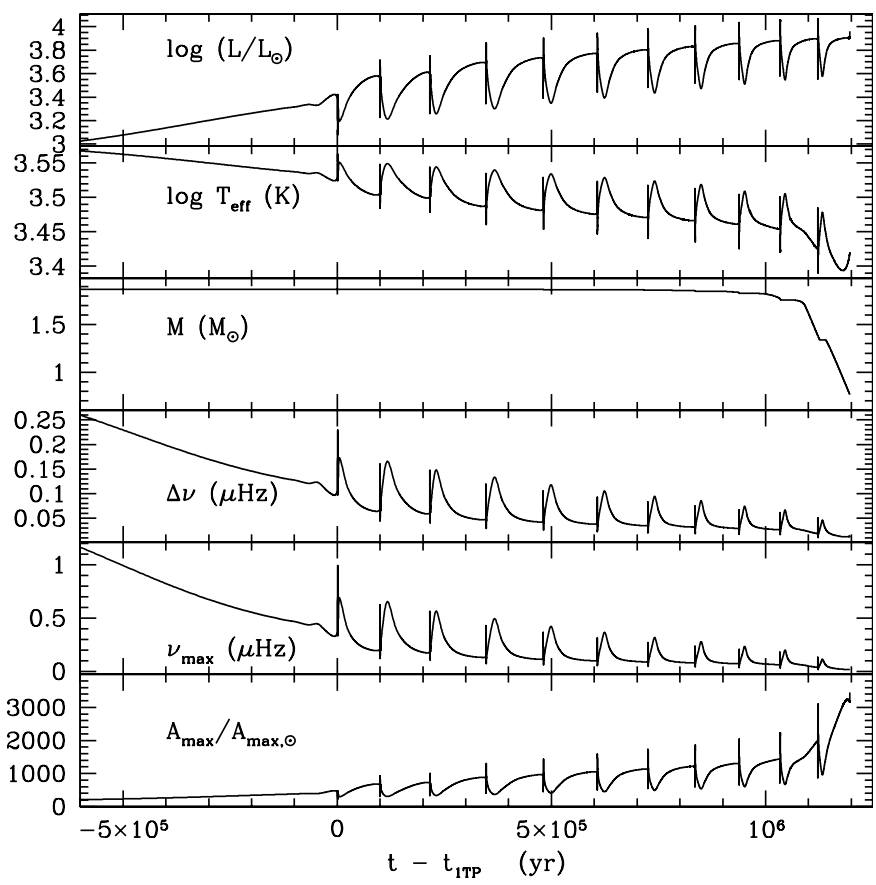

Fig. 10. From top to bottom: evolution of global stellar properties (luminosity $L$, effective temperature $T_{\text {eff }}$ and total stellar mass $M$ ) and asteroseismic parameters $\left(\Delta v\right.$, and $\left.v_{\max }, A_{\max } / A_{\max , \odot}\right)$ on the TP-AGB of the $\left[2.0 M_{\odot}, Z_{\odot}\right]$ model computed with rotation up to the RGB tip and thermohaline mixing all along the evolution. The abscissa is the time since the first thermal pulse.

the temperature, radius, luminosity, or mass of the models, this process has no impact on the global asteroseismic parameters. It is nevertheless not excluded that well-chosen asteroseismic parameters might help to constrain the thermohaline mixing.

As discussed in Sect. 4, rotation modifies the position of the evolutionary tracks in the HR diagram (see Fig. 2). Consequently, there are differences in both the maximal amplitude and the large separation, between the standard and rotating models. This effect clearly shows up at the end of the main sequence owing to the larger width of the main sequence in rotating models.

On the subgiant branch, rotating models then evolve to higher luminosities than their standard counterparts, which is indicative of a higher maximal amplitude. The rotation has no impact on the ratios $t_{\mathrm{BCE}} / T$ and $t_{\mathrm{He}} / T$ as seen in Fig. 8. In this figure, the effect of rotation on the stellar luminosity during the subgiant phase is also shown.

When the stars reach the RGB, the differences in effective temperature and luminosity between standard and rotating models become marginal, hence the tracks are almost identical in Fig. 6 (bottom left panel).

Rotation affects the extension and width of the blue loops. As a consequence, the differences in the asteroseismic parameters appear during the combustion phase of He, as illustrated in Fig. 6 (bottom right panel).

\subsection{Same position in HR-diagram, same asteroseismic parameters?}

Figure 11 shows how asteroseismic parameters can help us to distinguish between two stars with the same effective temperature and luminosity. In the left panel, we show the $1.5 M_{\odot}$ and 2.0 $M_{\odot}$ tracks in the HR diagram with the intersection indicated

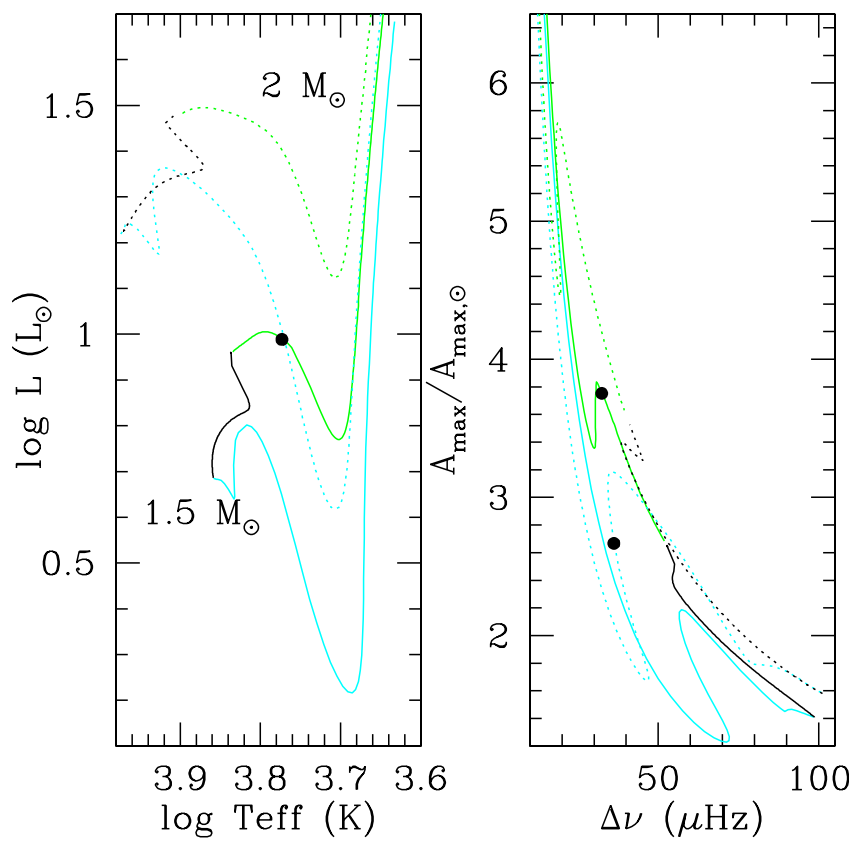

Fig. 11. Left panel: evolutionary tracks in Hertzsprung-Russell diagram of 1.5 and $2.0 Z_{\odot}$ (solid and dotted lines respectively) stars at solar metallicity. Right panel: maximal amplitude over Sun value as a function of the large separation for the same models. As in Fig. 5, the lines color indicates the evolutionary.

by the black dot. At this point, the $1.5 M_{\odot}$ star evolves within the HR gap and has the same effective temperature and luminosity as the $2.0 M_{\odot}$ star evolving on the pre-main sequence. This black dot corresponds to two different points in the $A_{\max }$ vs. $\Delta v$ plot shown in the right panel. As we can see, the larger separation of the $1.5 M_{\odot}$ model is smaller than that of the $2.0 M_{\odot}$ model, while the maximal amplitude is higher. These stars have neither the same mean stellar density, and nor same $\Delta v$. On the other hand, both the total stellar mass and the maximal amplitude are different at this point.

\section{Conclusions}

We have presented a grid of single-star evolution models in the mass range between $0.85 M_{\odot}$ and $6.0 M_{\odot}$, for four metallicities, including the impact of rotation and thermohaline mixing, along with standard models. All data detailed in Table 2 are available on a website ${ }^{9}$, for all the models computed (i.e., standard on the one hand, and with rotation-induced mixing and thermohaline instability on the other hand).

We recall the various impacts of metallicity variations and rotation-induced mixing on the stellar properties that were previously discussed in literature. Thermohaline mixing does not change stellar parameters such as luminosity and effective temperature, and indeed any of the seismic properties analyzed here. However and as discussed in Papers I and II, it changes the surface abundances from the bump luminosity on, and has to be taken into account to explain the observed chemical properties of bright giant stars.

Last but not least, we also present the evolution of global asteroseismic parameters for all the models in our grid. The large frequency separation $\Delta v$, the frequency $v_{\max }$, and the maximum oscillation amplitude $A_{\max }$ are computed using scaling relations. Asymptotic asteroseismic quantities are also computed,

${ }^{9}$ http://obswww. unige.ch/Recherche/evol/-Database- 
namely: $\Delta v_{\text {asympt }}$, another estimate or the large frequency separation; $t_{\mathrm{BCE}}$, the acoustic radius at the base of convective envelope; $t_{\mathrm{He}}$, the acoustic radius at the base of the HeII ionization zone; $T$, the total acoustic radius; and $\Delta \Pi(\ell=1)$, the period spacing of gravity modes.

We show that rotation-induced mixing has an impact on these quantities, in contrast to thermohaline mixing. While rotation changes the global properties of main sequence stars and has an impact on the global asteroseismic properties, thermohaline mixing is negligible in these aspects, although it does change the surface abundances in the red giants. In addition to spectrophotometric studies, seismic studies allow us to distinguish two stars with approximately the same luminosity and effective temperature but at different evolutionary stages.

Acknowledgements. We wish to thank the referee and Benoit Mosser for helpful comments on our manuscript. We acknowledge financial support from the Swiss National Science Foundation (FNS), from ESF-Euro Genesis, and the French Programme National de Physique Stellaire (PNPS) of CNRS/INSU.

\section{References}

Aikawa, M., Arnould, M., Goriely, S., Jorissen, A., \& Takahashi, K. 2005, A\&A, 441,1195

Arnould, M., Goriely, S., \& Jorissen, A. 1999, A\&A, 347, 572

Asplund, M., Grevesse, N., Sauval, A. J., \& Scott, P. 2009, ARA\&A, 47, 481

Bao, Z. Y., Beer, H., Käppeler, F., et al. 2000, in AIP Conf. Ser. 529 (Santa Fe,

New Mexico: AIP), 706

Beck, P. G., Bedding, T. R., Mosser, B., et al. 2011, Science, 332, 205

Bedding, T. R., Huber, D., Stello, D., et al. 2010, ApJ, 713, L176

Bedding, T. R., Mosser, B., Huber, D., et al. 2011, Nature, 471, 608

Beer, H., Sedyshev, P. V., Rochow, W., Mohr, P., \& Oberhummer, H. 2002, Nucl. Phys. A, 705, 239

Belkacem, K., Goupil, M. J., Dupret, M. A., et al. 2011, A\&A, 530, A142

Bildsten, L., Paxton, B., Moore, K., \& Macias, P. J. 2012, ApJ, 744, L6

Brott, I., de Mink, S. E., Cantiello, M., et al. 2011, A\&A, 530, A115

Brown, T. M., Gilliland, R. L., Noyes, R. W., \& Ramsey, L. W. 1991, ApJ, 368, 599

Brun, A. S., \& Palacios, A. 2009, ApJ, 702, 1078

Cantiello, M., \& Langer, N. 2010, A\&A, 521, A9

Cassisi, S., Pietrinferni, A., Salaris, M., et al. 2006, Mem. Soc. Astron. It., 77, 71

Caughlan, G. R., \& Fowler, W. A. 1988, At. Data Nucl. Data Tab., 40, 283 Chaboyer, B., \& Zahn, J.-P. 1992, A\&A, 253, 173

Chaplin, W. J., Appourchaux, T., Elsworth, Y., et al. 2010, ApJ, 713, L169

Chaplin, W. J., Kjeldsen, H., Bedding, T. R., et al. 2011a, ApJ, 732, 54

Chaplin, W. J., Kjeldsen, H., Christensen-Dalsgaard, J., et al. 2011b, Science,

332,213

Charbonnel, C. 1994, A\&A, 282, 811

Charbonnel, C., \& Lagarde, N. 2010, A\&A, 522, A10 (Paper I)

Charbonnel, C., \& Talon, S. 1999, A\&A, 351, 635

Charbonnel, C., \& Talon, S. 2005, Science, 309, 2189

Charbonnel, C., \& Talon, S. 2008, in IAU Symp. 252, eds. L. Deng, \& K. L. Chan (Sanya: CUP), 163

Charbonnel, C., \& Zahn, J. 2007a, A\&A, 476, L29

Charbonnel, C., \& Zahn, J.-P. 2007b, A\&A, 467, L15

Christensen-Dalsgaard, J. 2011 [arXiv: 1106.5946]

Coc, A., Vangioni-Flam, E., Descouvemont, P., Adahchour, A., \& Angulo, C. 2004, ApJ, 600, 544

Cunha, K., Hubeny, I., \& Lanz, T. 2006, ApJ, 647, L143

De Ridder, J., Barban, C., Baudin, F., et al. 2009, Nature, 459, 398

Decressin, T., Mathis, S., Palacios, A., et al. 2009, A\&A, 495, 271

Denissenkov, P. A. 2010, ApJ, 723, 563

Denissenkov, P. A., \& Merryfield, W. J. 2011, ApJ, 727, L8

Descouvemont, P., Adahchour, A., Angulo, C., Coc, A., \& Vangioni-Flam, E. 2004, At. Data Nucl. Data Tab., 88, 203

Dufour, E. 1999, Ph.D. Thesis, Université Joseph Fourier - Grenoble I

Eggenberger, P., Maeder, A., \& Meynet, G. 2005, A\&A, 440, L9

Eggenberger, P., Meynet, G., Maeder, A., et al. 2010a, A\&A, 519, A116

Eggenberger, P., Miglio, A., Montalban, J., et al. 2010b, A\&A, 509, A72
Eggleton, P. P., Faulkner, J., \& Flannery, B. P. 1973, A\&A, 23, 325

Eggleton, P. P., Dearborn, D. S. P., \& Lattanzio, J. C. 2006, Science, 314, 1580

Eggleton, P. P., Dearborn, D. S. P., \& Lattanzio, J. C. 2008, ApJ, 677, 581

Ekström, S., Georgy, C., Eggenberger, P., et al. 2012, A\&A, 537, A146

Ferguson, J. W., Alexander, D. R., Allard, F., et al. 2005, ApJ, 623, 585

Forestini, M., \& Charbonnel, C. 1997, A\&AS, 123, 241

Funck, C., \& Langanke, K. 1989, ApJ, 344, 46

Fynbo, H. O. U., Diget, C. A., Bergmann, U. C., et al. 2005, Nature, 433, 136

Graboske, H. C., Dewitt, H. E., Grossman, A. S., \& Cooper, M. S. 1973, ApJ, 181,457

Hale, S. E., Champagne, A. E., Iliadis, C., et al. 2002, Phys. Rev. C, 65, 015801

Hauser, W., \& Feshbach, H. 1952, Phys. Rev., 87, 366

Heger, A., \& Langer, N. 2000, ApJ, 544, 1016

Hekker, S., Kallinger, T., Baudin, F., et al. 2009, A\&A, 506, 465

Horiguchi, T., Tachibana, T., \& Katakura, J. 1996, Nuclear Data Center, Japan Atomic Energy Research Institute, Ibaraki

Huber, D., Bedding, T. R., Stello, D., et al. 2011, ApJ, 743, 143

Iglesias, C. A., \& Rogers, F. J. 1996, ApJ, 464, 943

Iliadis, C., D’Auria, J. M., Starrfield, S., Thompson, W. J., \& Wiescher, M. 2001, ApJS, 134, 151

Kawaler, S. D. 1988, ApJ, 333, 236

Kjeldsen, H., \& Bedding, T. R. 1995, A\&A, 293, 87

Koehler, P. E., \& O’brien, H. A. 1989a, Phys. Rev. C, 39, 1655

Koehler, P. E., \& O'brien, H. A. 1989b, Phys. Rev. C, 39, 1655

Krishnamurti, R. 2003, J. Fluid Mech., 483, 287

Lagarde, N., Charbonnel, C., Decressin, T., \& Hagelberg, J. 2011, A\&A, 536, A28 (Paper II)

Maeder, A. 1997, A\&A, 321, 134

Maeder, A. 2009, Physics, Formation and Evolution of Rotating Stars (Berlin Heidelberg: Springer)

Maeder, A., \& Meynet, G. 2000, ARA\&A, 38, 143

Maeder, A., \& Zahn, J.-P. 1998, A\&A, 334, 1000

Mathis, S., \& Zahn, J.-P. 2004, A\&A, 425, 229

Meynet, G., \& Maeder, A. 2000, A\&A, 361, 101

Michel, E., Baglin, A., Auvergne, M., et al. 2008, Science, 322, 558

Miglio, A., Montalbán, J., Baudin, F., et al. 2009, A\&A, 503, L21

Mitler, H. E. 1977, ApJ, 212, 513

Mosser, B., Belkacem, K., Goupil, M.-J., et al. 2010, A\&A, 517, A22

Mosser, B., Barban, C., Montalbán, J., et al. 2011, A\&A, 532, A86

Mukhamedzhanov, A. M., Bém, P., Brown, B. A., et al. 2003, Phys. Rev. C, 67, 065804

Palacios, A., Talon, S., Charbonnel, C., \& Forestini, M. 2003, A\&A, 399, 603

Palacios, A., Charbonnel, C., Talon, S., \& Siess, L. 2006, A\&A, 453, 261

Pols, O. R., Tout, C. A., Eggleton, P. P., \& Han, Z. 1995, MNRAS, 274, 964

Reimers, D. 1975, Mem. Soc. Roy. Sci. Liege, 8, 369

Rosenblum, E., Garaud, P., Traxler, A., \& Stellmach, S. 2011, ApJ, 731, 66

Schaller, G., Schaerer, D., Meynet, G., \& Maeder, A. 1992, A\&AS, 96, 269

Schaerer, D., Meynet, G., Maeder, A., \& Schaller, G. 1993, A\&AS, 98, 523

Schatz, H., Kaeppeler, F., Koehler, P. E., Wiescher, M., \& Trautvetter, H.-P. 1993, ApJ, 413, 750

Siess, L. 2009, A\&A, 497, 463

Siess, L., Dufour, E., \& Forestini, M. 2000, A\&A, 358, 593

Smiljanic, R., Pasquini, L., Charbonnel, C., \& Lagarde, N. 2010, A\&A, 510, A50

Stancliffe, R. J. 2010, MNRAS, 174

Stancliffe, R. J., Church, R. P., Angelou, G. C., \& Lattanzio, J. C. 2009, MNRAS 396,2313

Stello, D., Chaplin, W. J., Bruntt, H., et al. 2009, ApJ, 700, 1589

Talon, S., \& Charbonnel, C. 1998, A\&A, 335, 959

Talon, S., \& Charbonnel, C. 2003, A\&A, 405, 1025

Talon, S., \& Zahn, J.-P. 1997, A\&A, 317, 749

Traxler, A., Garaud, P., \& Stellmach, S. 2011, ApJ, 728, L29

Ulrich, R. K. 1971, ApJ, 168, 57

Ulrich, R. K. 1972, ApJ, 172, 165

Ulrich, R. K. 1986, ApJ, 306, L37

Uttenthaler, S., \& Lebzelter, T. 2010, A\&A, 510, A62

Vassiliadis, E., \& Wood, P. R. 1993, ApJ, 413, 641

Wagoner, R. V. 1969, ApJS, 18, 247

White, T. R., Bedding, T. R., Stello, D., et al. 2011, ApJ, 742, L3

Wiescher, M., Gorres, J., \& Thielemann, F. 1990, ApJ, 363, 340

Woosley, S. E., Fowler, W. A., Holmes, J. A., \& Zimmerman, B. A. 1978, At. Data Nucl. Data Tab., 22, 371

Yi, S. K., Kim, Y.-C., \& Demarque, P. 2003, ApJS, 144, 259

Zahn, J.-P. 1992, A\&A, 265, 115

Zahn, J.-P., Talon, S., \& Matias, J. 1997, A\&A, 322, 320 